\title{
FACTORES ABIÓTICOS INVOLUCRADOS EN LA FACILITACIÓN ENTRE LEÑOSAS Y SUCULENTAS EN EL ALTIPLANO MEXICANO
}

\author{
Rosa Romo-Campos ${ }^{1}$, José Luis Flores-Flores ${ }^{2}$, Joel Flores ${ }^{3}$ \\ y Gregorio Álvarez-Fuentes ${ }^{3}$ \\ 'Departamento de Ciencias Ambientales, Centro Universitario de Ciencias Biológicas y Agropecuarias, \\ Universidad de Guadalajara, Guadalajara, Jalisco, México \\ ${ }^{2}$ Instituto de Investigación de Zonas Desérticas, Universidad Autónoma de San Luis Potosí, \\ San Luis Potosí, San Luis Potosí, México \\ ${ }^{3}$ Instituto Potosino de Investigación Científica y Tecnológica, División de Ciencias Ambientales, \\ San Luis Potosí, San Luis Potosí, México \\ 'Autor para la correspondencia: rlrc.cucba@gmail.com
}

\begin{abstract}
Resumen: Se estudiaron características fisiológicas y morfológicas de plántulas de especies leñosas (nodrizas) y suculentas (protegidas), las cuales se distribuyen en un gradiente ambiental en el Altiplano Mexicano. Del extremo mésico se eligieron Acacia schaffneri, A. farnesiana y Opuntia jaliscana y, del extremo xérico se escogieron A. schaffneri, Prosopis laevigata y $O$. streptacantha. En un experimento en invernadero, plántulas de 24 semanas de edad fueron sometidas por 90 días a diferentes niveles de radiación (alta $=2133 \mu \mathrm{mol} \mathrm{m}^{-2} \mathrm{~d}^{-1}$, baja $\left.=166 \mu \mathrm{mol} \mathrm{m}^{-2} \mathrm{~d}^{-1}\right)$, humedad aprovechable (alta $=60 \%$, baja $\left.=30 \%\right)$ y fertilidad (alta $=$ suelo debajo de dosel, baja = suelo de área abierta). Las variables de respuesta evaluadas fueron la tasa relativa de crecimiento y sus componentes: morfológico (el cociente del área fotosintética) y fisiológico (la tasa de asimilación neta); así como la eficiencia cuántica del fotosistema II ( $\left.\Phi_{\mathrm{PSII}}\right)$ y la tasa de transporte de electrones. Las plántulas de suculentas presentaron mayor tasa relativa de crecimiento y cociente del área fotosintética que las plántulas de leñosas. La mayoría de las especies crecieron más en radiación y humedad altas. El suelo debajo de dosel favoreció el crecimiento de las suculentas, pero las leñosas presentaron los valores de la tasa de asimilación neta más altos. Los resultados sugieren que las plántulas de suculentas tienen mejor desempeño ecofisiológico para establecerse debajo del dosel de plantas nodrizas, y que las de leñosas presentan características ecofisiológicas de especies nodrizas mejor adaptadas a espacios abiertos.
\end{abstract}

Palabras clave: cociente del área fotosintética, eficiencia fotosintética, facilitación, tasa de asimilación neta, tasa de transporte de electrones, tasa relativa de crecimiento.

\begin{abstract}
We studied physiological and morphological characteristics of woody seedlings (nurses) and succulents (protected) which are distributed in an environmental gradient in the Mexican Plateau. Species from the mesic extreme of the gradient (Acacia schaffneri, A. farnesiana, Opuntia jaliscana) and from the xeric extreme (A. schaffneri, Prosopis laevigata, O. streptacantha) were chosen. 24-week-old seedlings were subjected for 90 days to varying levels of radiation (high $=2133 \mathrm{~mol} \mathrm{~m}^{-2} \mathrm{~d}^{-1}$, low $=166$ $\mathrm{mol} \mathrm{m}^{-2} \mathrm{~d}^{-1}$ ), available moisture (high $=60 \%$, low $=30 \%$ ) and fertility (high $=$ canopy soil, low $=$ open area). We assessed relative growth rate and its components: morphological (leaf area ratio) and physiological (net assimilation rate), as well as the quantum efficiency of photosystem II $\left(\Phi_{\mathrm{PSII}}\right)$, and the electron transport rate. Succulent seedlings had higher relative growth rate and leaf area ratio than woody ones. Most of the evaluated species grew more under high radiation and high humidity conditions. The canopy soil favored the growth of succulent species, but woody seedlings had the highest net assimilation rate. The results suggest that succulent seedlings showed ecophysiological functioning of protected species, best adapted to establish under the canopy of nurse plants. Woody species showed ecophysiological characteristics of nurses better adapted to open areas.

Keywords: Electron transport rate, facilitation, net assimilation rate, photosynthetic area ratio, quantum efficiency, relative growth rate.
\end{abstract}

A la influencia positiva de plantas adultas de una especie sobre la germinación, supervivencia y/o crecimiento de plántulas de otra especie se le conoce como "síndrome de planta nodriza" (Niering et al., 1963; Callaway, 1995) o interacciones "nodriza-protegida" (Cody, 1993). Este síndrome es muy importante en hábitats áridos y semiáridos (Franco y Nobel, 1989; Valiente-Banuet y Ezcurra, 1991; Flores y Jurado, 2003), donde es más frecuente que las 
plántulas de suculentas se establezcan debajo del dosel de plantas adultas de leñosas, a diferencia de las áreas abiertas (Valiente-Banuet et al., 1991; Flores-Martínez et al., 1994; Godínez-Álvarez et al., 2003; Flores y Jurado, 2003; Martínez-Berdeja y Valverde, 2008; Miquelajauregui y Valverde, 2010).

La sombra del dosel de las nodrizas protege a las plántulas contra la radiación extrema y por lo tanto, de una posible fotoinhibición (Nobel, 1980; Valiente-Banuet y Ezcurra, 1991; Cavender-Bares y Bazzaz, 2004). Además, al amortiguar la variación térmica, las plántulas transpiran menos y se disminuye la pérdida de humedad en el suelo (Domingo et al., 1999). El suelo bajo el dosel generalmente tiene mayor fertilidad debido a la acumulación de la hojarasca y materia orgánica (García-Moya y McKell 1970; Tirado y Pugnaire, 2003), lo que promueve el crecimiento de las plántulas (Miquelajauregui y Valverde, 2010).

Existen comunidades en las que todas las especies requieren de facilitación y, por lo tanto, ninguna es capaz de establecerse por sí misma, como en las Tetecheras de Neobuxbaumia mezcalensis del Valle de Tehuacán-Cuicatlán; sin embargo, en dos comunidades del mismo valle (Tetecheras de Neobuxbaumia tetetzo y Cardonales de Cephalocereus columna-trajani), la especie arbustiva Mimosa luisana se establece en espacios abiertos y al crecer, funciona como planta nodriza (Valiente-Banuet y Verdú, 2007). Otras especies nodrizas capaces de establecerse en espacios abiertos son Ambrosia dummosa en el Desierto Sonorense (McAuliffe, 1988), Prosopis glandulosa en matorrales del sur de Texas (Brown y Archer, 1987; Bush y Van Auken, 1987; Barnes y Archer, 1999) y P. laevigata en el Desierto Chihuahuense, donde la ganadería extensiva crea claros en los pastizales permitiendo su entrada a la comunidad vegetal (Flores y Yeaton, 2000). Las diferencias en el crecimiento entre grupos de especies son debidas a cambios temporales en los factores ambientales a las que están adaptadas, y pueden indicar la capacidad de adaptación a su nicho de regeneración. Por ejemplo, las nodrizas maximizan el uso de nutrimentos y agua en sitios expuestos, y las protegidas en micrositios debajo del dosel de leñosas adultas, donde la sombra aumenta su supervivencia y crecimiento (Gonçalvez et al., 2005).

Muchas variables ambientales entre sitios expuestos y protegidos son diferentes, por lo que se esperaría que nodrizas y protegidas tuvieran diferencias en sus requerimientos de luz, humedad y fertilidad (Lambers et al., 1998; Butterfield y Briggs, 2011). Estas variaciones se reflejan en su tasa relativa de crecimiento, que es clave para conocer la función ecológica de las especies (Grime y Hunt, 1975; Poorter y Garnier, 1999). Dichas diferencias están en función de características fisiológicas, morfológicas y genéticas (Poorter y Garnier, 1999). Por ejemplo, las especies tolerantes a altas radiaciones (las nodrizas), generalmente presentan alta tasa relativa de crecimiento, así como tasa de asimilación neta y cociente raíz/vástago altos, debido a que el factor dominante en su crecimiento es la luz (Poorter y Van der Werf, 1998). En cambio, las tolerantes a la sombra (e.g. las protegidas) tienen crecimiento bajo (Grime, 1977; Lambers y Poorter, 1992), cociente del área foliar alto y cociente raíz/vástago bajo (Poorter, 1999), debido a que el factor dominante para su crecimiento es la sombra.

Adicionalmente, la fluorescencia de la clorofila es una herramienta que puede ser utilizada para analizar el estrés en plantas bajo diferentes ambientes (Cavender-Bares y Bazzaz, 2004), como los que están presentes para las plántulas de nodrizas y de protegidas. Por ejemplo, la radiación alta puede afectar las reacciones fotoquímicas de la fotosíntesis en las especies que crecen en las áreas abiertas, lo que disminuye su eficiencia fotosintética potencial y actual (Osmond, 1994). Sin embargo, la tasa de transporte de electrones aumenta (Cavender-Bares y Bazzaz, 2004).

Para las especies que participan en las interacciones de facilitación es muy importante el efecto simultáneo de la luz, humedad y nutrimentos sobre el crecimiento de nodrizas y protegidas (Martínez-Berdeja y Valverde, 2008). Sin embargo; no se ha investigado como influyen los factores abióticos en la respuesta fisiológica de especies nodrizas y protegidas en etapa de plántulas. El propósito de este trabajo fue evaluar la respuesta ecofisiológica de plántulas de tres especies de leñosas y dos suculentas a condiciones contrastantes relacionadas con el microambiente de protección o fuera de éste. La hipótesis es que las leñosas (nodrizas) tienen respuestas fisiológicas y morfológicas de especies adaptadas a espacios abiertos (radiación alta, humedad y fertilidad bajas); y las suculentas (protegidas), al contario de las anteriores, presentan adaptaciones a micrositios con sombra. Son pocas las publicaciones que comparan interacciones entre especies de ecosistemas áridos y mésicos, por lo que este trabajo puede proporcionar conocimientos en torno a los cambios en la estructura y dinámica de las comunidades vegetales que se distribuyen a través de gradientes de productividad y precipitación (Butterfield y Briggs, 2011).

\section{Materiales y métodos}

Sitios de colecta de semillas. La región se localiza en el centro-occidente de México. La vegetación dominante es el matorral xerófilo con plantas arbustivas y suculentas llamadas localmente "nopaleras" (Miranda y Hernández, 1963), típicas de zonas áridas y semiáridas (Rzedowski, 1966; INEGI, 2002). Las semillas de las especies estudiadas (leñosas y suculentas), se recolectaron de dos sitios en los extremos de un gradiente ambiental en el centro-occidente de México. En el extremo seco, al noreste del gradiente, está el rancho Santiago, en Villa de Arriaga, San Luis Potosí ( $21^{\circ} 55^{\prime} 25.67$ ” N; $101^{\circ} 13$ ' 53.98" O y 2,363 m s.n.m.). El material geológico son rocas ígneas extrusivas y terrenos planos alternados con lomeríos suaves, con pendientes entre 5 y $10 \%$. El suelo 
predominante es el xerosol, de color claro y pobre en materia orgánica. La precipitación pluvial promedio anual es de $375.5 \mathrm{~mm}$. El clima es semiseco templado BS1kw(e), con lluvias en verano y con oscilación térmica extrema $\left(1.8^{\circ} \mathrm{C}\right.$ mínima y $29.3{ }^{\circ} \mathrm{C}$ máxima), la temperatura media anual es de $12.5^{\circ} \mathrm{C}$. La temporada de lluvias ocurre entre los meses de julio a octubre (INEGI, 2002).

El extremo con mayor humedad, al suroeste del gradiente, es el rancho Los Vallejo en Jalostotitlán, Jalisco ( $21^{\circ} 10^{\prime}$ 48.57” N; $102^{\circ} 28^{\prime} 38.30^{\prime \prime}$ O, a 1,772 m s.n.m.). Su sustrato son rocas sedimentarias, con lomeríos de pendientes entre 10 y $25 \%$. Sus suelos son predominantes del tipo feozem háplico, oscuros y ricos en materia orgánica, poco profundos y muy rocosos. La precipitación pluvial promedio anual es de $703 \mathrm{~mm}$ y la temperatura media anual de $17.9^{\circ} \mathrm{C}$, con máxima y mínima de $37.4{ }^{\circ} \mathrm{C}$ y $-2.5^{\circ} \mathrm{C}$, respectivamente. El clima es cálido-subhúmedo $\mathrm{Ca}(\mathrm{w} 0)(\mathrm{w})(\mathrm{e})$, con lluvias en verano y con oscilación térmica extrema, la precipitación invernal es menor al 5\% de la total (INEGI, 1981).

Especies seleccionadas. En cada sitio se eligieron dos especies leñosas y una suculenta. En el sitio xérico (Villa de Arriaga, San Luis Potosí), las leñosas fueron Acacia schaffneri (S.Wats.) F.J.Herm., Prosopis laevigata Humb. \& Bonpl. ex Willd, y la suculenta Opuntia streptacantha Lemaire. En el sitio mésico (Jalostotitlán, Jalisco), fueron $A$. farnesiana (L.) Willd., A. schaffneri y $O$. jaliscana Bravo. En este trabajo, las leñosas serán nombradas como nodrizas y las suculentas como protegidas.

Crecimiento de las plántulas en invernadero. Se colectaron semillas de diez individuos adultos por especie y sitio en agosto de 2007. Después de remover la pulpa de los frutos, las semillas fueron guardadas en bolsas de papel estraza, a temperatura ambiente y en condiciones de oscuridad por seis meses. En abril de 2008 las semillas fueron escarificadas para romper la latencia (Reyes-Agüero et al., 2005; Silveria y Fernandes, 2006). Doscientas semillas de cada especie fueron sembradas en macetas con suelo natural esterilizado térmicamente a $105{ }^{\circ} \mathrm{C}$, mezclado con peat moss (1:1). Las semillas germinaron a los nueve días después de la siembra. A los 45 días se seleccionaron 75 plántulas por especie y fueron trasplantadas a bolsas de plástico de $35 \times$ $15 \times 20 \mathrm{~cm}$ (una plántula por bolsa). Cada bolsa se llenó con $4 \mathrm{~kg}$ de suelo procedente de los micrositios (abierto y bajo el dosel de Acacia schaffneri, como niveles de fertilidad) procedente de cada área.

Se utilizó el diseño de tratamientos factorial $2 \times 2 \times 2$ $\times 6$ con los siguientes factores y niveles: luz (alta y baja, con sombra inducida mediante una malla al $75 \%$ ); humedad aprovechable (baja y alta, 30 y $60 \%$, respectivamente); fertilidad del suelo (baja y alta: suelo abierto y suelo de dosel, respectivamente; de Jalostotitlán (mésico) y de Villa de Arriaga (xérico); y las especies Acacia schaffneri del sitio mésico, A. schaffneri del sitio xérico, A. farnesiana, Prosopis laevigata, Opuntia jaliscana y $O$. streptacantha (se tienen seis niveles debido a dos procedencias de A. schaffneri).

El diseño experimental para fines operativos fue de parcelas subdivididas en bloques al azar con tres repeticiones. La parcela grande correspondió a los niveles de luz y la parcela chica a los niveles de humedad. La fertilidad y las especies se asignaron al azar dentro de las parcelas chicas. Los niveles de humedad se calcularon a partir de la capacidad de campo y del punto de marchitez permanente de cada suelo por micrositio para obtener la humedad aprovechable. Para el seguimiento y control de los niveles de humedad se utilizó el método gravimétrico y un dispositivo reflectrométrico (TDR, Campbell, modelo Hydrosense). El nivel alto de radiación correspondió a exposición total al sol y el bajo a una reducción del $75 \%$ con malla plástica. En el tratamiento de exposición a luz alta, el promedio del PAR a las 9:00 a.m. fue de $1200 \mu \mathrm{mol} \mathrm{m}^{-2} \mathrm{~s}^{-1}$, y en el tratamiento de luz baja fue de $230 \mu \mathrm{mol} \mathrm{m}^{-2} \mathrm{~s}^{-1}$.

Variables de respuesta. La tasa relativa de crecimiento (TRC) se evaluó mediante dos cosechas (30 y 90 días) (Lambers y Poorter, 1992). El peso fresco de las plántulas en la primera cosecha se usó para corregir los pesos de las macetas y controlar los niveles de humedad entre la primera y la segunda cosecha. Tres plántulas por especie fueron cosechadas, órganos aéreos y raíces fueron secados en una estufa a $70{ }^{\circ} \mathrm{C}$ por 48 horas para obtener su biomasa. El área fotosintética de leñosas y suculentas se obtuvo digitalizando imágenes en fresco con un escáner marca HP G3010. Las imágenes se analizaron con el software Sigma Scan/image (Jandel Scientific, 1993).

La TRC y sus componentes (el cociente del área fotosintética y la tasa de asimilación neta), se calcularon de acuerdo a Cardillo y Bernal (2006), según la tasa relativa de crecimiento (TRC, $\mathrm{mg}^{-1} \mathrm{~g}^{-1}$ día $^{-1}$ ), que mide los cambios en la biomasa de las plántulas a través del tiempo y puede ser dividida en dos componentes: el morfológico (CAF, cociente de área fotosintética, en $\mathrm{cm}^{2} \mathrm{~g}^{-1}$ ), es el total del área fotosintética por unidad de biomasa total y, el componente fisiológico (TAN, tasa de asimilación neta, en $\mathrm{mg} / \mathrm{día} / \mathrm{cm}^{2}$ ), que mide la asignación de biomasa resultado de la diferencia entre la tasa fotosintética neta diaria y la respiración (Evans, 1972).

$\left.\left.\operatorname{TRC}=\left(\left(B T_{2}-B T_{1}\right) /\left(T_{2}-T_{1}\right)\right) *((2))\left(B T_{1}+B T_{2}\right)\right) / T A N=\left(\left(B T_{2}-B T_{1}\right) /\left(T_{2}-T_{1}\right)\right) *\left((2) / A F T_{1}+A F T_{2}\right)\right)$

Donde: $\mathrm{BT}_{1}$ y $\mathrm{BT}_{2}$ son la biomasa promedio de las plantas en las cosechas 1 y $2 ; \mathrm{T}_{1}$ y $\mathrm{T}_{2}$ son el tiempo inicial y final de las dos cosechas; $\mathrm{AFT}_{1} \mathrm{y} \mathrm{AFT}_{2}$, son el total del área fotosintética en la primera y segunda cosecha. La TRC también se puede obtener mediante el producto de CAF*TAN (Cardillo y Bernal, 2006). 
El cociente raíz/vástago se obtuvo del cociente de la biomasa de las raíces entre la de los vástagos y no tiene unidades. Los valores mayores a la unidad indican que las plántulas asignan más biomasa a la raíz que a la parte aérea (Miquelajauregui y Valverde, 2010).

La eficiencia fotosintética actual (EFA) del $\Phi_{\text {PSII }}(\Delta F /$ $F_{\mathrm{m}}$ ), en una muestra adaptada a la luz, se calculó mediante la fórmula:

$$
\Delta F / F_{\mathrm{m}},=\left(F_{\mathrm{m}},-F\right) / F_{\mathrm{m}},
$$

La eficiencia fotosintética potencial (EFP) del $\Phi_{\text {PSII }}$ en una muestra adaptada a la oscuridad se calculó con la fórmula:

$$
F_{v} / F_{\mathrm{m}},
$$

Donde $F_{v}$ es la fluorescencia de la muestra adaptada a la oscuridad. $F$ es la fluorescencia de la clorofila de la muestra adaptada a la luz, y $F_{\mathrm{m}}$, es la fluorescencia máxima de la muestra adaptada a la luz cuando se aplica un pulso de saturación de luz actínica (Maxwell y Johnson, 2000; CavenderBares y Bazzaz, 2004). Estas variables se midieron en la segunda cosecha a las 5:00 a.m. (pre-amanecer) y a las 12:00 p.m. (mediodía), con un analizador portátil de fluorescencia (Mini-PAM, Photosynthesis Yield Analyzer, Walz, Alemania). La tasa de transporte de electrones (TTE; Ehleringer, 1981):

TTE $=$ EFA del $\Phi_{\text {PSII }} \times$ RFA $\times 0.05 \times 0.084$, expresado en $\mu \mathrm{mol} \mathrm{m} \mathrm{m}^{-2} \mathrm{~s}^{-1}$
EFA del $\Phi_{\text {PSII }}$ es la eficiencia fotosintética actual del fotosistema II, RFA es la radiación fotosintéticamente activa, 0.05 es un factor de distribución de energía entre el fotosistema I $\left(\Phi_{\text {PSI }}\right)$ y el fotosistema II $\left(\Phi_{\text {PSII }}\right)$, y 0.84 es un factor estándar de la fracción de luz absorbida por el tejido fotosintético.

Análisis estadísticos. Los atributos relevantes de fertilidad de los suelos (como la materia orgánica y el nitrógeno amoniacal), de los diferentes micrositios de cada sitio se analizaron mediante ANOVA de una vía. Los valores de la tasa relativa de crecimiento se obtuvieron mediante las nueve combinaciones posibles con las tres repeticiones obtenidas de la primera cosecha, con las respectivas medidas de la segunda cosecha; ésta es una modificación del método que sólo considera la comparación de tres pares de valores cuando se tienen tres repeticiones. De esta manera se generaron nueve valores de los que se obtuvo un promedio por tratamiento. Este procedimiento permitió disminuir la variación de los resultados, comparado con el procedimiento habitual de calcular la TRC con sólo un par de datos por repetición.

La tasa relativa de crecimiento (TRC) y sus componentes se analizaron con un ANOVA multifactorial, de acuerdo con el diseño factorial utilizado previamente, con el software SAS (SAS, 2002). Este diseño fue útil para un mejor manejo de los tratamientos; sin embargo, debido a que se encontraron diferencias entre sitios $\mathrm{y}$, dentro de los sitios para los grupos funcionales, los análisis de varianza se realizaron por especie. Para las variables que no cumplieron

Cuadro 1. Promedio \pm EE de los atributos de suelos por micrositio: (debajo del dosel de Acacia schaffneri y en abierto) y por localidad: rancho

\begin{tabular}{|c|c|c|c|c|c|}
\hline \multirow{2}{*}{\multicolumn{2}{|c|}{ Suelo }} & \multicolumn{2}{|c|}{ Sitio mésico } & \multicolumn{2}{|c|}{ Sitio xérico } \\
\hline & & \multirow{2}{*}{$\begin{array}{l}\text { Dosel } \\
56.9 \pm 1.7\end{array}$} & \multirow{2}{*}{$\begin{array}{l}\text { Abierto } \\
64.6 \pm 0.9\end{array}$} & \multirow{2}{*}{$\begin{array}{l}\text { Dosel } \\
84.5 \pm 0.6\end{array}$} & \multirow{2}{*}{$\begin{array}{l}\text { Abierto } \\
73.58 \pm 0.7\end{array}$} \\
\hline Textura & arena & & & & \\
\hline & limo & $35.3 \pm 0.88$ & $25.3 \pm 1.3$ & $12 \pm 0.6$ & $12 \pm 2.0$ \\
\hline & arcilla & $8 \pm 2.1$ & $11.1 \pm 1.1$ & $3.5 \pm 0.001$ & $14.42 \pm 2.7$ \\
\hline \multirow{2}{*}{\multicolumn{2}{|c|}{$\begin{array}{c}\text { Materia orgánica \% } \\
\mathrm{pH}\end{array}$}} & $12.1 \pm 3.4$ & $2.8 \pm 0.4$ & $6.64 \pm 1.3$ & $2.25 \pm 0.4$ \\
\hline & & $5.13 \pm 0.02$ & $5.19 \pm 0.01$ & $5.9 \pm 0.4$ & $4.85 \pm 0.6$ \\
\hline \multicolumn{2}{|c|}{ Capacidad de intercambio catiónico } & $47.33 \pm 6.4$ & $16.1 \pm 1.14$ & $30.25 \pm 4.4$ & $10.29 \pm 0.4$ \\
\hline \multirow[t]{5}{*}{ Cationes Intercambiables } & $\mathrm{Ca}+\mathrm{Mg} \mathrm{Meq} / 100 \mathrm{~g}$ & $8.46 \pm 1.4$ & $3.78 \pm 0.26$ & $7.03 \pm 1.8$ & $2.86 \pm 0.9$ \\
\hline & Ca meq/10 g & $6.64 \pm 1.4$ & $2.47 \pm 0.1$ & $5.21 \pm 1.3$ & $1.56 \pm 0.4$ \\
\hline & Mg meq/100 g & $1.83 \pm 0.1$ & $1.31 \pm 0.1$ & $1.83 \pm 0.57$ & $1.76 \pm 0.3$ \\
\hline & Na meq/100 g & $0.88 \pm 0.001$ & $0.09 \pm 0.02$ & $0.15 \pm 0.02$ & $0.066 \pm 0.01$ \\
\hline & $\mathrm{K}$ meq/100 g & $1.09 \pm 0.05$ & $0.67 \pm 0.08$ & $1.03 \pm 0.14$ & $0.445 \pm 0.6$ \\
\hline \multirow[t]{7}{*}{ Fertilidad } & $\mathrm{N}-\mathrm{NO}_{3} \mathrm{ppm}$ & $18.5 \pm 0.001$ & $6 \pm 0.001$ & $22.8 \pm 0.001$ & $3.0 \pm 0.001$ \\
\hline & $\mathrm{N}-\mathrm{NH}_{4} \mathrm{ppm}$ & $150 \pm 0.001$ & $72.5 \pm 7.5$ & $182 \pm 0.001$ & $35 \pm 0.001$ \\
\hline & P ppm & $25 \pm 0.001$ & $25 \pm 0.001$ & $25 \pm 0.17$ & $25 \pm 0.001$ \\
\hline & K ppm & $226.6 \pm 2$ & $191.7 \pm 11.7$ & $250 \pm 0.1$ & $250 \pm 0.001$ \\
\hline & Ca ppm & $1333 \pm 1$ & $833 \pm 66.7$ & $1400 \pm 11$ & $500 \pm 0.001$ \\
\hline & Mg ppm & $25 \pm 0.001$ & $25 \pm 0.001$ & $25 \pm 0.1$ & $18.5 \pm 3.7$ \\
\hline & Mn ppm & $22.5 \pm 10$ & $5 \pm 0.001$ & $15 \pm 5.7$ & $5 \pm 0.001$ \\
\hline
\end{tabular}
Los Vallejo, Jalostotitlán, Jalisco (sitio mésico) y rancho Santiago, Villa de Arriaga, San Luis Potosí (sitio xérico) $(\mathrm{n}=3)$. 
los supuestos para el análisis en ANOVA, los datos se transformaron a la escala $\log$ natural, particularmente se aplicó el análisis Kruskall-Wallis en el CAF y la TAN de Opuntia jaliscana, por no cumplir dichos supuestos. El grado de asociación entre la TRC y sus componentes se realizó mediante un análisis de regresión. Los datos de la fluorescencia de la clorofila se analizaron con MANOVA, debido a que las variables EFA, TTE y el PAR presentaron interdependencia. Para efectos de notación en los cuadros de resultados se abreviarán los factores por sus iniciales: radiación $=\mathrm{R}$, humedad $=\mathrm{H}$ y fertilidad $=\mathrm{F}$; asimismo, el nivel mayor se denotará como alto y el menor como bajo.

\section{Resultados}

El análisis fisicoquímico de los suelos por localidad y micrositio, indicó diferencias para algunas variables (Cuadro 1). La materia orgánica fue diferente entre micrositios $(F=$ $115.82, P<0.01)$, el suelo debajo de dosel del sitio mésico presentó $77 \%$ más materia orgánica, comparado con el suelo en abierto del mismo sitio; además, el suelo de dosel del sitio xérico presentó $67 \%$ más materia orgánica que el suelo en abierto del mismo sitio. También hubo diferencia significativa en la interacción de sitio $\times$ micrositio $(F=21.54, P$ $<0.01)$. El suelo debajo de dosel del sitio mésico presentó cerca del doble de materia orgánica que el del sitio xérico.

Tasa relativa de crecimiento (TRC, $\mathrm{mg}^{-1} \mathrm{~g}^{-1}$ día $^{-1}$ ). Para el gradiente total (ambos sitios), se encontró que las especies del sitio mésico crecieron $18 \%$ más que las especies del sitio xérico (mésico: $0.020 \pm 0.00055$, xérico: $0.017 \pm 0.0007$ ). Las protegidas tuvieron en promedio $22 \%$ mayor TRC que las nodrizas (Opuntia jaliscana: $0.022 \pm 0.0006$, O. streptacantha: 0.023 \pm 0.0007 , Acacia schaffneri (sitio mésico): $0.020 \pm 0.0008$, A. farnesiana: $0.017 \pm 0.0008$, Prosopis laevigata: $0.016 \pm$ 0.0008 y A. schaffneri (sitio xérico): $0.015 \pm 0.0014$ ).

Dentro de cada sitio también se observaron diferencias entre nodrizas. En el sitio mésico, Acacia farnesiana presentó $14 \%$ menor crecimiento que A. schaffneri (A. farnesiana: $0.0176 \pm 0.0008$, A. schaffneri de Jalostotitlán: $0.0206 \pm 0.0008$ ), mientras que en el sitio xérico las leñosas presentaron prácticamente la misma TRC (Prosopis laevigata: $0.0157 \pm 0.0008$, A. schaffneri: $0.0155 \pm 0.001$ ).

La respuesta por especie a los tratamientos fue diferente, como se especifica a continuación. Acacia farnesiana presentó diferencias sólo para el factor fertilidad $(F=5.61, P=$ $<0.05$ ), la fertilidad alta disminuyó su crecimiento en $17 \%$, en contraste con la fertilidad baja. En A. schaffneri del sitio mésico, la humedad alta aumentó la TRC en $16 \%$ al compararla con la humedad baja $(F=10.56, P=<0.01)$; mientras que la radiación y la fertilidad no fueron significativas. Además, el efecto de la humedad fue distinto por el nivel de fertilidad; la humedad alta y la fertilidad baja aumentaron el crecimiento en $28 \%$, en comparación con la humedad y fertilidad bajas ( $F=8.93, P=<0.01$; Cuadro 2).

Acacia schaffneri del sitio xérico en la radiación alta creció $31 \%$ más que en la baja y, en la humedad alta la TRC aumentó $28 \%$ más al compararla con la humedad baja, (Luz: $F=7.88, P=<0.01$; humedad $F=5.9, P=0.02$, respectivamente). El factor fertilidad no presentó efectos significativos. Sin embargo, el efecto de la fertilidad fue distinto por el nivel de radiación, la radiación alta y la fertilidad baja aumentaron su crecimiento en promedio $43 \%$, en comparación con las demás combinaciones (Cuadro 3).

En Prosopis laevigata la TRC aumentó en 17\% en la radiación alta, en comparación con la radiación baja, y 17.2\% en la humedad baja en contraste con la humedad alta $(F=$ 8.07, $P=<0.05 ; F=5.82, P=<0.05$, respectivamente; Cuadro 2). El efecto de la humedad fue distinto por el nivel de radiación, en el tratamiento de radiación alta y humedad baja la TRC aumentó en promedio un 30\% (Cuadro 3).

La TRC de Opuntia jaliscana aumentó $13.1 \%$, 13\% y $15 \%$ como respuesta a la radiación, humedad y fertilidad altas, al compararlos con la radiación, humedad y fertilidad bajas $(F=120.31, P=<0.01 ; F=118.83, P=<0.01$; $F=298.51, P=<0.01$, respectivamente; Cuadro 2). Todas las interacciones dobles fueron significativas, el efecto de la humedad fue distinto por el nivel de radiación, la radiación y la humedad altas incrementaron la TRC en promedio $16 \%$. El efecto de la fertilidad fue distinto por el nivel de radiación: la radiación baja y la fertilidad baja disminuyeron el crecimiento en promedio $26.6 \%$. El efecto de la fertilidad fue diferente por el nivel de humedad: la humedad y la fertilidad altas aumentaron en $25 \%$ el crecimiento en contraste con el de humedad y fertilidad bajas (Cuadro 3).

Las plántulas de Opuntia streptacantha presentaron diferencias estadísticas significativas en los factores de radiación, humedad y fertilidad $(F=27.24, P=<<0.01 ; F=9.41$, $P=<0.01 ; F=21.06, P=<0.01$, respectivamente; Cuadro 2). La radiación, humedad y fertilidad altas aumentaron la TRC en $8 \%, 12.5 \%$ y $12 \%$, respectivamente; en comparación con la radiación, humedad y fertilidad bajas. También se encontraron interacciones significativas. El efecto de la humedad fue distinto por el nivel de radiación: en la radiación alta y la humedad alta aumentó el crecimiento en 19\%, en contraste con la radiación y humedad bajas. El efecto de la fertilidad también fue diferente por el nivel de radiación: en la radiación baja y la fertilidad baja disminuyó la TRC en $19 \%$, comparado con la radiación y fertilidad altas. La fertilidad varió por el nivel de humedad: la humedad alta y la fertilidad alta aumentó la TRC en $22 \%$ en contraste con la humedad baja y fertilidad alta (Cuadro 3). Además, se presentaron diferencias en la interacción triple radiación, humedad y fertilidad, el efecto de la humedad y la fertilidad fueron distintos por el nivel de la radiación: la radiación, humedad y fertilidad altas aumentó el crecimiento en 35\%, en comparación con la radiación baja, humedad alta y fertilidad baja (Cuadro 3). 
Cuadro 2. Para tres plantas nodrizas y dos protegidas: promedios $( \pm \mathrm{EE}) \times 10^{-2}$ de la tasa relativa de crecimiento $\left(\mathrm{mg}^{-1} \mathrm{~g}-1 \mathrm{dí}^{-1}\right)$. Promedios $( \pm \mathrm{EE})$ del cociente del área fotosintética $\left(\mathrm{cm}^{2} \mathrm{~g}^{-1}\right)$. Promedios $( \pm \mathrm{EE}) \times 10^{-3}$ de la tasa de asimilación neta $\left(\mathrm{mg} / \mathrm{día} / \mathrm{cm}^{2}\right)$ y $( \pm \mathrm{EE})$ del cociente raíz/vástago. Las letras en negritas denotan promedios con diferencia significativa $(P<0.05)$.

\begin{tabular}{|c|c|c|c|c|c|}
\hline Especie & Nivel de radiación & $\begin{array}{l}\text { Tasa relativa de } \\
\text { crecimiento }\end{array}$ & $\begin{array}{l}\text { Cociente del área } \\
\text { fotosintética }\end{array}$ & $\begin{array}{c}\text { Tasa de asimilación } \\
\text { neta }\end{array}$ & $\begin{array}{l}\text { Cociente raíz/ } \\
\text { vástago }\end{array}$ \\
\hline \multirow[t]{2}{*}{ Acacia farnesiana } & alta & $0.0185 \pm 0.001^{\mathrm{ns}}$ & $7.8 \pm 0.19^{b}$ & $0.0023 \pm 0.0001^{\mathrm{a}}$ & $0.77 \pm 0.04^{\mathrm{ns}}$ \\
\hline & baja & $0.0176 \pm 0.001^{\mathrm{ns}}$ & $9.3 \pm 0.2^{\mathrm{a}}$ & $0.0017 \pm 0.0001^{b}$ & $0.76 \pm 0.05^{\mathrm{ns}}$ \\
\hline \multirow{2}{*}{$\begin{array}{l}\text { Acacia schaffneri } \\
\text { del sitio mésico }\end{array}$} & alta & $0.022 \pm 0.001^{\mathrm{ns}}$ & $6.1 \pm 0.2^{b}$ & $0.0034 \pm 0.0002^{\mathrm{a}}$ & $1.4 \pm 0.05^{b}$ \\
\hline & baja & $0.020 \pm 0.001^{\mathrm{ns}}$ & $7.8 \pm 0.2^{\mathrm{a}}$ & $0.0020 \pm 0.0001^{b}$ & $1.1 \pm 0.04^{a}$ \\
\hline \multirow{2}{*}{$\begin{array}{c}\text { Acacia schaffneri } \\
\text { del sitio xérico }\end{array}$} & alta & $0.018 \pm 0.002^{\mathrm{a}}$ & $4.6 \pm 0.2^{b}$ & $0.0041 \pm 0.0003^{a}$ & $1.9 \pm 0.07^{b}$ \\
\hline & baja & $0.013 \pm 0.002^{b}$ & $7.5 \pm 0.3^{a}$ & $0.0015 \pm 0.0002^{b}$ & $1.3 \pm 0.05^{a}$ \\
\hline \multirow[t]{2}{*}{ Prosopis laevigata } & alta & $0.017 \pm 0.002^{\mathrm{a}}$ & $4.5 \pm 0.25^{b}$ & $0.0034 \pm 0.0002^{a}$ & $0.7 \pm 0.05^{b}$ \\
\hline & baja & $0.014 \pm 0.001^{b}$ & $7.3 \pm 0.26^{a}$ & $0.002 \pm 0.0001^{b}$ & $0.45 \pm 0.03^{a}$ \\
\hline \multirow[t]{2}{*}{ Opuntia jaliscana } & alta & $0.023 \pm 0.0006^{a}$ & $12.4 \pm 0.2$ & $0.0021 \pm 0.00006$ & $0.24 \pm 0.02^{\mathrm{ns}}$ \\
\hline & baja & $0.020 \pm 0.001^{b}$ & $19.7 \pm 0.3$ & $0.001 \pm 0.00003$ & $0.21 \pm 0.02^{\mathrm{ns}}$ \\
\hline \multirow[t]{2}{*}{ Opuntia streptacantha } & alta & $0.023 \pm 0.0009^{a}$ & $13.7 \pm 0.5^{b}$ & $0.0017 \pm 0.0001^{\mathrm{a}}$ & $0.18 \pm 0.02^{\mathrm{ns}}$ \\
\hline & baja & $0.021 \pm 0.0011^{b}$ & $19.5 \pm 0.3^{\mathrm{a}}$ & $0.001 \pm 0.00005^{b}$ & $0.2 \pm 0.02^{\mathrm{ns}}$ \\
\hline \multirow[t]{2}{*}{ Acacia farnesiana } & alta & $0.019 \pm 0.001^{\mathrm{ns}}$ & $8.5 \pm 0.2^{\mathrm{ns}}$ & $0.002 \pm 0.0001^{\mathrm{ns}}$ & $0.77 \pm 0.04^{\mathrm{ns}}$ \\
\hline & baja & $0.018 \pm 0.001^{\mathrm{ns}}$ & $8.7 \pm 0.3^{\mathrm{ns}}$ & $0.002 \pm 0.0002^{\mathrm{ns}}$ & $0.76 \pm 0.04^{\mathrm{ns}}$ \\
\hline \multirow{2}{*}{$\begin{array}{l}\text { Acacia schaffneri } \\
\text { del sitio mésico }\end{array}$} & alta & $0.023 \pm 0.0007^{a}$ & $6.9 \pm 0.4^{\mathrm{ns}}$ & $0.003 \pm 0.0004^{\mathrm{ns}}$ & $1.4 \pm 0.05^{b}$ \\
\hline & baja & $0.019 \pm 0.001^{b}$ & $7.0 \pm 0.2^{\mathrm{ns}}$ & $0.0025 \pm 0.0001^{\mathrm{ns}}$ & $1.1 \pm 0.04^{a}$ \\
\hline \multirow{2}{*}{$\begin{array}{c}\text { Acacia schaffneri } \\
\text { del sitio xérico }\end{array}$} & alta & $0.018 \pm 0.001^{\mathrm{a}}$ & $6.3 \pm 0.4^{\mathrm{ns}}$ & $0.003 \pm 0.0003^{a}$ & $1.9 \pm 0.07^{b}$ \\
\hline & baja & $0.013 \pm 0.0021^{b}$ & $5.9 \pm 0.5^{\mathrm{ns}}$ & $0.0026 \pm 0.0006^{b}$ & $1.3 \pm 0.05^{a}$ \\
\hline \multirow[t]{2}{*}{ Prosopis laevigata } & alta & $0.015 \pm 0.0007^{a}$ & $5.9 \pm 0.4^{\mathrm{ns}}$ & $0.0026 \pm 0.0002^{\mathrm{ns}}$ & $0.66 \pm 0.05^{b}$ \\
\hline & baja & $0.017 \pm 0.001^{b}$ & $5.9 \pm 0.6^{\mathrm{ns}}$ & $0.0027 \pm 0.0003^{\mathrm{ns}}$ & $0.45 \pm 0.03^{a}$ \\
\hline \multirow[t]{2}{*}{ Opuntia jaliscana } & alta & $0.023 \pm 0.001^{\mathrm{a}}$ & $15.9 \pm 1.1^{\mathrm{ns}}$ & $0.0016 \pm 0.0001^{\mathrm{ns}}$ & $0.24 \pm 0.02^{\mathrm{ns}}$ \\
\hline & baja & $0.020 \pm 0.0006^{b}$ & $16.1 \pm 1.1^{\mathrm{ns}}$ & $0.0016 \pm 0.0002^{\mathrm{ns}}$ & $0.21 \pm 0.03^{\mathrm{ns}}$ \\
\hline \multirow[t]{2}{*}{ Opuntia streptacantha } & alta & $0.024 \pm 0.001^{\mathrm{a}}$ & $16.5 \pm 1.1^{\mathrm{ns}}$ & $0.0016 \pm 0.0001^{a}$ & $0.20 \pm 0.01^{b}$ \\
\hline & baja & $0.021 \pm 0.0003^{b}$ & $16.7 \pm 0.9^{\mathrm{ns}}$ & $0.0013 \pm 0.0001^{b}$ & $0.17 \pm 0.01^{a}$ \\
\hline \multirow[t]{2}{*}{ Acacia farnesiana } & alta & $0.016 \pm 0.001^{b}$ & $8.0 \pm 0.2^{a}$ & $0.0018 \pm 0.0001^{b}$ & $0.6 \pm 0.01^{a}$ \\
\hline & baja & $0.02 \pm 0.0008^{a}$ & $9.1 \pm 0.2^{b}$ & $0.0023 \pm 0.0001^{a}$ & $0.9 \pm 0.03^{b}$ \\
\hline \multirow{2}{*}{$\begin{array}{l}\text { Acacia schaffneri } \\
\text { del sitio mésico }\end{array}$} & alta & $0.021 \pm 0.001^{\mathrm{ns}}$ & $7.1 \pm 0.3^{\mathrm{ns}}$ & $0.0026 \pm 0.0003^{\mathrm{ns}}$ & $1.1 \pm 0.05^{b}$ \\
\hline & baja & $0.021 \pm 0.0007^{\mathrm{ns}}$ & $6.7 \pm 0.3^{\mathrm{ns}}$ & $0.0028 \pm 0.0002^{\mathrm{ns}}$ & $1.3 \pm 0.07^{a}$ \\
\hline \multirow{2}{*}{$\begin{array}{c}\text { Acacia schaffneri } \\
\text { del sitio xérico }\end{array}$} & alta & $0.017 \pm 0.002^{\mathrm{ns}}$ & $6.0 \pm 0.4^{\mathrm{ns}}$ & $0.0024 \pm 0.0005^{b}$ & $1.58 \pm 0.07^{b}$ \\
\hline & baja & $0.014 \pm 0.001^{\mathrm{ns}}$ & $6.1 \pm 0.2^{\mathrm{ns}}$ & $0.0031 \pm 0.0004^{a}$ & $1.73 \pm 0.1^{\mathrm{a}}$ \\
\hline \multirow[t]{2}{*}{ Prosopis laevigata } & alta & $0.016 \pm 0.001^{\mathrm{ns}}$ & $6.7 \pm 0.5^{\mathrm{a}}$ & $0.0025 \pm 0.0002^{\mathrm{ns}}$ & $0.45 \pm 0.04^{b}$ \\
\hline & baja & $0.016 \pm 0.001^{\mathrm{ns}}$ & $5.1 \pm 0.5^{b}$ & $0.0029 \pm 0.0003^{\mathrm{ns}}$ & $0.67 \pm 0.04^{a}$ \\
\hline \multirow[t]{2}{*}{ Opuntia jaliscana } & alta & $0.024 \pm 0.0006^{a}$ & $16.2 \pm 0.9^{\text {ns }}$ & $0.0016 \pm 0.0002^{a}$ & $0.16 \pm 0.01^{b}$ \\
\hline & baja & $0.020 \pm 0.0006^{b}$ & $15.8 \pm 1.3^{\mathrm{ns}}$ & $0.0015 \pm 0.0002^{b}$ & $0.3 \pm 0.01^{\mathrm{a}}$ \\
\hline \multirow[t]{2}{*}{ Opuntia streptacantha } & alta & $0.024 \pm 0.0009^{a}$ & $17.7 \pm 1.1^{\mathrm{a}}$ & $0.0015 \pm 0.0001^{\mathrm{ns}}$ & $0.18 \pm 0.01^{b}$ \\
\hline & baja & $0.021 \pm 0.001^{b}$ & $15.6 \pm 0.7^{b}$ & $0.0015 \pm 0.00021^{\mathrm{ns}}$ & $0.2 \pm 0.01^{\mathrm{a}}$ \\
\hline
\end{tabular}


Cuadro 3. Resultados de ANOVA para la tasa relativa de crecimiento (TRC). Interacciones entre factores de estudio: $\mathrm{R}=\mathrm{Radiación}, \mathrm{H}=\mathrm{Humedad}$ y $\mathrm{F}=$ Fertilidad. Se presentan el cuadrado medio $(\mathrm{CM})$, valor de $F$ y valor de significancia para $P(<0.05)$ en letras negritas.

\begin{tabular}{|c|c|c|c|c|c|c|c|c|c|c|c|c|c|c|c|c|c|c|}
\hline \multirow[b]{2}{*}{ TRC } & \multicolumn{3}{|c|}{$\begin{array}{c}\text { Acacia } \\
\text { farnesiana }\end{array}$} & \multicolumn{3}{|c|}{$\begin{array}{l}\text { Acacia schaffneri } \\
\text { del sitio mésico }\end{array}$} & \multicolumn{3}{|c|}{$\begin{array}{l}\text { Acacia schaffneri } \\
\text { del sitio xérico }\end{array}$} & \multicolumn{3}{|c|}{$\begin{array}{l}\text { Prosopis } \\
\text { laevigata }\end{array}$} & \multicolumn{3}{|c|}{$\begin{array}{l}\text { Opuntia } \\
\text { jaliscana }\end{array}$} & \multicolumn{3}{|c|}{$\begin{array}{c}\text { Opuntia } \\
\text { streptacantha }\end{array}$} \\
\hline & $\begin{array}{c}\text { Efectos } \\
\text { CM } \\
* 10^{-6}\end{array}$ & $F$ & $\begin{array}{l}\text { Valor } \\
\text { de } P\end{array}$ & $\begin{array}{c}\text { Efectos } \\
\text { CM } \\
* 10^{-6}\end{array}$ & $F$ & $\begin{array}{l}\text { Valor } \\
\text { de } P\end{array}$ & $\begin{array}{c}\text { Efectos } \\
\text { CM } \\
* 10^{-6}\end{array}$ & $F$ & $\begin{array}{l}\text { Valor } \\
\text { de } P\end{array}$ & $\begin{array}{c}\text { Efectos } \\
\text { CM } \\
* 10^{-6}\end{array}$ & $F$ & $\begin{array}{l}\text { Valor } \\
\text { de } P\end{array}$ & $\begin{array}{c}\text { Efectos } \\
\text { CM } \\
* 10^{-6}\end{array}$ & $F$ & $\begin{array}{l}\text { Valor } \\
\text { de } P\end{array}$ & $\begin{array}{c}\text { Efectos } \\
\text { CM } \\
* 10^{-6}\end{array}$ & $F$ & $\begin{array}{l}\text { Valor } \\
\text { de } P\end{array}$ \\
\hline $\mathrm{R}^{*} \mathrm{H}$ & 5.37 & 0.45 & 0.51 & 1.9 & 0.28 & 0.6 & 1.11 & 0.05 & 0.83 & 43.9 & 4.75 & 0.04 & 1.92 & 5.91 & 0.027 & 18.6 & 8.65 & 0.01 \\
\hline $\mathrm{R} * \mathrm{~F}$ & 1.5 & 0.12 & 0.72 & 13.6 & 2.02 & 0.17 & 146.1 & 6.01 & 0.02 & 0.52 & 0.06 & 0.82 & 31.7 & 97.78 & 0.001 & 17.2 & 8.0 & 0.01 \\
\hline $\mathrm{H}^{*} \mathrm{~F}$ & 24.4 & 2.03 & 0.17 & 60.4 & 8.93 & 0.01 & 14.5 & 0.64 & 0.43 & 2.51 & 0.27 & 0.61 & 15.5 & 47.73 & 0.001 & 59.44 & 27.65 & 0.001 \\
\hline $\mathrm{R}^{*} \mathrm{H}^{*} \mathrm{~F}$ & 3.8 & 0.32 & 0.57 & 6.38 & 0.94 & 0.35 & 60.5 & 2.49 & 0.13 & 52.5 & 5.68 & 0.03 & 0.2 & 0.66 & 0.43 & 23.66 & 11.01 & 0.004 \\
\hline
\end{tabular}

Cociente del área fotosintética $\left(\mathrm{CAF}, \mathrm{cm}^{2} \mathrm{~g}^{-1}\right)$. Se utiliza área fotosintética en lugar de área foliar por estar incluidas especies suculentas, donde hay área fotosintética y no hojas propiamente dichas. Los resultados indican que el CAF no varió entre sitios (sitio mésico: $10.53 \pm 0.54$, sitio xérico: $9.54 \pm 0.65$ ), pero sí entre especies, dado que las nodrizas mostraron menor CAF $(6.9 \pm 0.18)$ que las protegidas $(16.3$ \pm 0.51 ). Dentro de cada sitio, las nodrizas del sitio mésico aumentaron su CAF en promedio $26.5 \%$ con respecto a las del sitio xérico. El CAF entre protegidas no varió.

El CAF aumentó en todas las especies como respuesta a la luz baja; sin embargo, respondieron de forma diferente. Acacia farnesiana presentó diferencias significativas en los factores de radiación $(F=127.29, P=<0.01)$ y fertilidad $(F=44.76, P=<0.01)$. En la radiación baja el CAF aumentó $16 \%$ en comparación con la radiación alta, mientras que en la fertilidad alta el CAF fue $10 \%$ mayor que en fertilidad baja. Los niveles de humedad no presentaron diferencias (Cuadro 2). También se encontraron diferencias en la interacción radiación y humedad, en todas sus combinaciones; la radiación y la humedad bajas incrementó el CAF en 9.2\%, $17.24 \%$ y $23 \%$ en comparación con los de radiación baja y humedad alta, radiación y humedad altas y, la radiación alta y humedad baja, respectivamente (Cuadro 4).

Acacia schaffneri del sitio mésico presentó diferencia estadística solamente para el factor radiación $(F=79.15$,
$P=<0.01)$, en la radiación baja el CAF aumentó $23.6 \%$ comparado con el de la radiación alta (Cuadro 2). En A. schaffneri del sitio xérico también se hallaron diferencias para el factor radiación $(F=585.94, P=<0.01)$, el CAF en la radiación baja fue $39 \%$ mayor que el encontrado en la radiación alta (Cuadro 2). Todas las interacciones dobles fueron significativas (Cuadro 4). La interacción triple reveló que el CAF más bajo se encontró por efecto de la radiación alta, humedad baja y fertilidad alta (Cuadro 4).

La radiación baja aumentó el CAF de Prosopis laevigata en $93 \%$ con respecto al de la radiación alta, mientras que en la fertilidad alta lo aumentó en $23 \%$ comparado con el de la fertilidad baja $(F=366.84, P=<0.01 ; F=101.79, P=<0.01$; respectivamente). No hubo efectos por los niveles de humedad (Cuadro 2). Sin embargo, el efecto de la humedad fue distinto por el nivel de radiación: en la radiación y humedad bajas el CAF aumentó 8.5\% en comparación con el de la radiación y humedad altas (Cuadro 4).

Para el CAF en Opuntia jaliscana se encontraron diferencias entre los niveles de radiación con el análisis KruskalWallis ( $H=17.28, P=<0.01)$, el CAF fue $63 \%$ mayor en luz baja que en luz alta $(19.7 \pm 0.2,12.4 \pm 0.33$; respectivamente). La humedad y la fertilidad no fueron diferentes (Cuadro 2). Opuntia streptacantha presentó diferencias significativas en los factores de radiación y fertilidad $(F=307.98, P=<0.1$; $F=41.33, P=<0.1$; respectivamente). En la radiación baja

Cuadro 4. Resultados de ANOVA para el cociente del área fotosintética (CAF). Interacciones entre factores de estudio: $\mathrm{R}=\mathrm{Radiación}, \mathrm{H}=\mathrm{Hu}$ medad y $\mathrm{F}=$ Fertilidad. Se presentan el cuadrado medio $(\mathrm{CM})$, valor de $F$ y valor de significancia para $P(<0.05)$ en letras negritas.

\begin{tabular}{|c|c|c|c|c|c|c|c|c|c|c|c|c|c|c|c|c|c|c|}
\hline \multirow[b]{2}{*}{ CAF } & \multicolumn{3}{|c|}{$\begin{array}{c}\text { Acacia } \\
\text { farnesiana }\end{array}$} & \multicolumn{3}{|c|}{$\begin{array}{l}\text { Acacia schaffneri } \\
\text { del sitio mésico }\end{array}$} & \multicolumn{3}{|c|}{$\begin{array}{l}\text { Acacia schaffneri } \\
\text { del sitio xérico }\end{array}$} & \multicolumn{3}{|c|}{$\begin{array}{l}\text { Prosopis } \\
\text { laevigata }\end{array}$} & \multicolumn{3}{|c|}{$\begin{array}{l}\text { Opuntia } \\
\text { jaliscana }\end{array}$} & \multicolumn{3}{|c|}{$\begin{array}{c}\text { Opuntia } \\
\text { streptacantha }\end{array}$} \\
\hline & $\begin{array}{c}\text { Efectos } \\
\text { CM }\end{array}$ & $F$ & $\begin{array}{l}\text { Valor } \\
\text { de } P\end{array}$ & $\begin{array}{c}\text { Efectos } \\
\text { CM }\end{array}$ & $F$ & $\begin{array}{l}\text { Valor } \\
\text { de } P\end{array}$ & $\begin{array}{c}\text { Efectos } \\
\text { CM }\end{array}$ & $F$ & $\begin{array}{l}\text { Valor } \\
\text { de } P\end{array}$ & $\begin{array}{l}\text { Efectos } \\
\text { CM }\end{array}$ & $F$ & $\begin{array}{l}\text { Valor } \\
\text { de } P\end{array}$ & $\begin{array}{c}\text { Efectos } \\
\text { CM }\end{array}$ & $F$ & $\begin{array}{l}\text { Valor } \\
\text { de } P\end{array}$ & $\begin{array}{c}\text { Efectos } \\
\text { CM }\end{array}$ & $F$ & $\begin{array}{l}\text { Valor } \\
\text { de } P\end{array}$ \\
\hline $\mathrm{R}^{*} \mathrm{H}$ & 3.15 & 29.59 & 0.001 & 2.9 & 11.9 & 0.003 & 3.608 & 43.06 & 0.001 & 1.72 & 12.85 & 0.002 & 0.44 & 1.23 & 0.28 & 2.49 & 3.85 & 0.07 \\
\hline $\mathrm{R} * \mathrm{~F}$ & 0.049 & 0.46 & 0.5 & 0.05 & 0.22 & 0.64 & 2.198 & 26.22 & 0.001 & 0.00657 & 0.05 & 0.83 & 9.31 & 26.01 & 0.001 & 7.58 & 11.73 & 0.01 \\
\hline $\mathrm{H}^{*} \mathrm{~F}$ & 0.035 & 0.33 & 0.57 & 0.61 & 2.48 & 0.135 & 1.366 & 16.3 & 0.001 & 0.138 & 1.03 & 0.326 & 2.5 & 7.0 & 0.017 & 1.02 & 1.58 & 0.23 \\
\hline $\mathrm{R} * \mathrm{H} * \mathrm{~F}$ & 0.033 & 0.32 & 0.58 & 0.09 & 0.36 & 0.55 & 4.53 & 54.11 & 0.001 & 0.19 & 1.42 & 0.25 & 0.452 & 1.26 & 0.28 & 0.434 & 0.67 & 0.42 \\
\hline
\end{tabular}


Rosa Romo-CAmpos ET AL.

Cuadro 5. Resultados de ANOVA para la tasa de asimilación neta (TAN). Interacciones entre factores de estudio: $\mathrm{R}=\mathrm{Radiación}, \mathrm{H}=\mathrm{Humedad}$ y $\mathrm{F}=$ Fertilidad. Se presentan el cuadrado medio $(\mathrm{CM})$, valor de $F$ y valor de significancia para $P(<0.05)$ en letras negritas.

\begin{tabular}{|c|c|c|c|c|c|c|c|c|c|c|c|c|c|c|c|}
\hline \multirow[b]{2}{*}{ TRC } & \multicolumn{3}{|c|}{$\begin{array}{c}\text { Acacia } \\
\text { farnesiana }\end{array}$} & \multicolumn{3}{|c|}{$\begin{array}{l}\text { Acacia schaffneri } \\
\text { del sitio mésico }\end{array}$} & \multicolumn{3}{|c|}{$\begin{array}{l}\text { Acacia schaffneri } \\
\text { del sitio xérico }\end{array}$} & \multicolumn{3}{|c|}{$\begin{array}{l}\text { Prosopis } \\
\text { laevigata }\end{array}$} & \multicolumn{3}{|c|}{$\begin{array}{c}\text { Opuntia } \\
\text { streptacantha }\end{array}$} \\
\hline & $\begin{array}{c}\text { Efectos } \\
\text { CM } \\
* 10^{-7}\end{array}$ & $F$ & $\begin{array}{l}\text { Valor } \\
\text { de } P\end{array}$ & $\begin{array}{c}\text { Efectos } \\
\text { CM } \\
* 10^{-7}\end{array}$ & $F$ & $\begin{array}{l}\text { Valor } \\
\text { de } P\end{array}$ & $\begin{array}{c}\text { Efectos } \\
\text { CM } \\
* 10^{-7}\end{array}$ & $F$ & $\begin{array}{l}\text { Valor } \\
\text { de } P\end{array}$ & $\begin{array}{c}\text { Efectos } \\
\text { CM } \\
* 10^{-7}\end{array}$ & $F$ & $\begin{array}{l}\text { Valor } \\
\text { de } P\end{array}$ & $\begin{array}{c}\text { Efectos } \\
\text { CM } \\
* 10^{-7}\end{array}$ & $F$ & $\begin{array}{l}\text { Valor } \\
\text { de } P\end{array}$ \\
\hline $\mathrm{R} * \mathrm{H}$ & 0.004 & 0.11 & 0.74 & 43.9 & 151.5 & 0.001 & 19.2 & 15.65 & 0.001 & 4.9 & 1.55 & 0.23 & 4.23 & 54.96 & 0.001 \\
\hline $\mathrm{R}^{*} \mathrm{~F}$ & 1.37 & 3.39 & 0.084 & 4.6 & 15.8 & 0.001 & 10.8 & 8.79 & 0.009 & 2.2 & 0.71 & 0.41 & 1.7 & 22.09 & 0.002 \\
\hline $\mathrm{H}^{*} \mathrm{~F}$ & 19.4 & 47.9 & 0.001 & 5.0 & 17.4 & 0.001 & 41.8 & 34.0 & 0.001 & 4.7 & 1.48 & 0.24 & 3.82 & 49.62 & 0.001 \\
\hline $\mathrm{R}^{*} \mathrm{H}^{*} \mathrm{~F}$ & 2.22 & 5.48 & 0.032 & 13.7 & 47.2 & 0.001 & 61.5 & 50.08 & 0.001 & 0.0 & 0.00 & 0.99 & 0.142 & 1.84 & 0.19 \\
\hline
\end{tabular}

el CAF aumentó $30 \%$ en comparación con el de la radiación alta, mientras que en la fertilidad alta presentó $13 \%$ más CAF que en el de la fertilidad baja (Cuadro 2). Los niveles de humedad no fueron estadísticamente distintos. Además, la combinación de la radiación baja y fertilidad alta incrementó el CAF en 39\%, comparado con el de radiación alta y fertilidad baja (Cuadro 4).

Tasa de asimilación neta (TAN, mg/día/cm2). En promedio, la TAN en el sitio xérico fue de $0.0024 \pm 0.00017$ y en el sitio mésico fue de $0.0022 \pm 0.0001$. Las nodrizas presentaron una TAN de $0.0027 \pm 0.0 .0001 \mathrm{y}$ las protegidas de $0.0014 \pm 0.00007$. Todas las especies aumentaron la TAN por efecto de la luz alta; sin embargo, dicho aumento fue diferente en cada especie. En Acacia farnesiana se encontró que la TAN aumentó $23.5 \%$ en la radiación alta en comparación con el de radiación baja y, en el tratamiento de fertilidad alta disminuyó $23 \%$ en contraste con el de fertilidad baja $(F=39.97, P=<0.01 ; F=41.25, P=<0.01$; respectivamente; Cuadro 2). El efecto de la humedad fue distinto por el nivel de fertilidad, la TAN aumentó $42 \%$ en el tratamiento de humedad y fertilidad bajas, al compararlo con el de humedad baja y fertilidad alta. Además, el efecto de la humedad y el de la fertilidad fue distinto por el nivel de radiación, la mayor TAN se presentó en el tratamiento de radiación alta, humedad baja y fertilidad alta; mientras que la menor fue en el de radiación baja, humedad alta y fertilidad baja (Cuadro 5).

Para Acacia schaffneri del sitio mésico en los factores de radiación, humedad y fertilidad hubo diferencias estadísticas $(F=409.63, P=<0.01 ; F=34.07, P=<0.01$; $F=9.46, P=<0.01$, respectivamente), en contraste con los tratamientos de radiación, humedad y fertilidad bajas, la TAN aumentó $41 \%$ en presencia de radiación alta, $15 \%$ en humedad alta y $7 \%$ en fertilidad alta (Cuadro 2). También se presentaron diferencias en todas las interacciones. En el tratamiento de radiación alta y humedad alta, la TAN fue $55 \%$ superior que la que se encontró en el de radiación y humedad bajas. Esta disminuyó en $40 \%$ y $21 \%$ por efecto de la radiación y fertilidad bajas y, en el de la humedad y fertilidad bajas, respectivamente (Cuadro 2). El efecto de la humedad y la fertilidad fueron diferentes por el nivel de la radiación; la TAN fue $67.5 \%$ mayor en el de radiación alta, humedad alta y fertilidad baja, en comparación con el de radiación baja, humedad alta y fertilidad baja (Cuadro5).

En Acacia schaffneri del sitio xérico la TAN aumentó $73 \%, 17 \%$ y $22.5 \%$ en los tratamientos de radiación alta, humedad alta y fertilidad baja; en contraste con los de radiación baja, humedad baja y fertilidad alta; respectivamente $(F=319.5, P=<0.01 ; F=10.6 . P=<0.01 ; F=26.3, P=<$ 0.01 ; respectivamente) (Cuadro 2). En las todas las interacciones se encontraron diferencias. La TAN aumentó $75 \%$ en

Cuadro 6. Resultados de ANOVA para el cociente raíz/vástago $(\mathrm{C}: \mathrm{R} / \mathrm{V})$. Interacciones entre factores de estudio: $\mathrm{R}=\mathrm{Radiación}, \mathrm{H}=\mathrm{Humedad}$ y $\mathrm{F}=$ Fertilidad. Se presentan el cuadrado medio $(\mathrm{CM})$, valor de $F$ y valor de significancia para $P(<0.05)$ en letras negritas.

\begin{tabular}{|c|c|c|c|c|c|c|c|c|c|c|c|c|c|c|c|c|c|c|}
\hline \multirow[b]{2}{*}{$C: R / V$} & \multicolumn{3}{|c|}{$\begin{array}{c}\text { Acacia } \\
\text { farnesiana }\end{array}$} & \multicolumn{3}{|c|}{$\begin{array}{l}\text { Acacia schaffneri } \\
\text { del sitio mésico }\end{array}$} & \multicolumn{3}{|c|}{$\begin{array}{l}\text { Acacia schaffneri } \\
\text { del sitio xérico }\end{array}$} & \multicolumn{3}{|c|}{$\begin{array}{l}\text { Prosopis } \\
\text { laevigata }\end{array}$} & \multicolumn{3}{|c|}{$\begin{array}{l}\text { Opuntia } \\
\text { jaliscana }\end{array}$} & \multicolumn{3}{|c|}{$\begin{array}{c}\text { Opuntia } \\
\text { streptacantha }\end{array}$} \\
\hline & $\begin{array}{c}\text { Efectos } \\
\text { CM } \\
* 10^{-3}\end{array}$ & $F$ & $\begin{array}{l}\text { Valor } \\
\text { de } P\end{array}$ & $\begin{array}{c}\text { Efectos } \\
\text { CM } \\
* 10^{-3}\end{array}$ & $F$ & $\begin{array}{l}\text { Valor } \\
\text { de } P\end{array}$ & $\begin{array}{c}\text { Efectos } \\
\text { CM } \\
* 10^{-2}\end{array}$ & $F$ & $\begin{array}{l}\text { Valor } \\
\text { de } P\end{array}$ & $\begin{array}{c}\text { Efectos } \\
\text { CM } \\
* 10^{-2}\end{array}$ & $F$ & $\begin{array}{l}\text { Valor } \\
\text { de } P\end{array}$ & $\begin{array}{l}\text { Efectos } \\
\text { CM } \\
* 10^{-3}\end{array}$ & $F$ & $\begin{array}{l}\text { Valor } \\
\text { de } P\end{array}$ & $\begin{array}{c}\text { Efectos } \\
\text { CM } \\
* 10^{-3}\end{array}$ & $F$ & $\begin{array}{l}\text { Valor } \\
\text { de } P\end{array}$ \\
\hline $\mathrm{R}^{*} \mathrm{H}$ & 17.05 & 3.57 & 0.08 & 15.2 & 1.11 & 0.31 & 5.56 & 3.38 & 0.084 & 5.7 & 21.7 & 0.003 & 0.001 & 0.0 & 0.98 & 24.2 & 84.9 & 0.001 \\
\hline $\mathrm{R}^{*} \mathrm{~F}$ & 0.29 & 0.06 & 0.81 & 31.4 & 2.3 & 0.15 & 48.01 & 29.2 & 0.001 & 0.44 & 1.69 & 0.21 & 3.07 & 1.53 & 0.23 & 7.11 & 24.9 & 0.001 \\
\hline $\mathrm{H}^{*} \mathrm{~F}$ & 11.1 & 2.33 & 0.14 & 32.8 & 2.41 & 0.14 & 0.94 & 0.57 & 0.46 & 2.6 & 9.85 & 0.006 & 1.71 & 0.86 & 0.37 & 0.003 & 0.01 & 0.92 \\
\hline $\mathrm{R} * \mathrm{H}^{*} \mathrm{~F}$ & 0.16 & 0.03 & 0.65 & 0.77 & 0.06 & 0.81 & 0.415 & 0.25 & 0.62 & 1.74 & 6.62 & 0.02 & 10.4 & 5.18 & 0.037 & 0.57 & 1.99 & 0.177 \\
\hline
\end{tabular}


el tratamiento de radiación y humedad altas, comparado con el de radiación baja y humedad baja. Sin embargo, disminuyó $70 \%$ en el de radiación baja y fertilidad alta, en contraste con el de radiación alta y fertilidad baja, y se redujo $45 \%$ en el de humedad y fertilidad altas a diferencia con el de humedad baja y fertilidad baja. El efecto de la humedad y la fertilidad fue diferente por el nivel de radiación, en el de radiación alta, humedad alta y fertilidad baja hubo una TAN 66\% mayor que el de radiación baja, humedad baja y fertilidad alta (Cuadro 5).

Prosopis laevigata sólo presentó efectos significativos en el factor luz $(F=41.54, P=<0.01)$. En la radiación alta la TAN aumentó en $47 \%$ en comparación con el de radiación baja. El análisis de Kruskal-Wallis para Opuntia jaliscana reveló que la TAN fue 52\% mayor en el de radiación alta que en el de radiación baja $(H=17.28, P=<0.01)$, los niveles de humedad y fertilidad no fueron distintos (Cuadro 2). Opuntia streptacantha por efecto de la radiación alta aumentó $66 \%$ su TAN en comparación con la radiación baja; mientras que por la humedad alta la TAN fue $19 \%$ mayor que en la humedad baja $(F=365, P=<0.01 ; F=72.55, P=<0.01$; respectivamente). Los niveles de fertilidad no presentaron diferencias (Cuadro 2). Se encontraron diferencias estadísticas en las siguientes interacciones: en el tratamiento de radiación alta y humedad alta, la TAN aumentó $48 \%$ en contraste con el de radiación y humedad bajas. El efecto de la fertilidad fue distinto por el nivel de luz en todas sus combinaciones, en la radiación alta y fertilidad alta se presentó una TAN 29\% mayor que el de radiación y fertilidad bajas, y en el tratamiento de humedad alta y fertilidad alta fue $31 \%$ mayor en contraste con el de humedad y fertilidad bajas (Cuadro 5).

Cociente Raíz/Vástago (C:R/V). El C:R/V varió entre sitios, en el mésico fue $9 \%$ más alto que en el xérico $(0.74 \pm 0.05$, $0.8 \pm 0.08$; respectivamente). Entre grupos funcionales se halló que las nodrizas presentaron $80 \%$ más $\mathrm{C}: \mathrm{R} / \mathrm{V}$ que las protegidas $(1.05 \pm 0.5,0.21 \pm 0.01$; respectivamente). El C: $\mathrm{R} / \mathrm{V}$ de las nodrizas fue superior o cercano a la unidad, lo cual indica que éstas especies asignaron más biomasa a la raíz que al tallo, resultados contrarios se encontraron en las protegidas con valores de $\mathrm{C}: \mathrm{R} / \mathrm{V}$ cercanos al cero, revelando que asignaron más biomasa al tallo que a la raíz.

En todas las especies, el suelo de fertilidad baja aumentó el C:R/V. Para Acacia farnesiana la fertilidad baja aumentó el C:R/V en $28 \%$ en comparación con el nivel alto $(F=88.29, P=<0.01)$; la radiación y la humedad no presentaron efectos significativos (Cuadro 2). Para $A$. schaffneri del sitio mésico, el nivel de radiación alto y el de fertilidad bajo aumentaron el C:R/V en $24 \%$ y $16 \%$, correspondientemente, al compararlos con el de radiación baja y el de fertilidad alta $(F=44.73, P=<0.01 ; F=17.16$, $P=<0.01$, respectivamente; Cuadro 2). En A. schaffneri del sitio xérico como respuesta a la radiación alta, el C:R/V aumentó $31 \%$ ( $F=134.32, P=<0.01)$, comparado con el de la radiación baja. Además, la fertilidad baja incrementó el C:R/V en $8.7 \%$. Los niveles de humedad no presentaron diferencias (Cuadro 2). También se encontraron diferencias en el tratamiento de radiación y fertilidad altas, este aumentó el C:R/V en $80 \%$ en contraste con el de radiación alta y fertilidad baja (Cuadro 6).

Prosopis laevigata presentó diferencias por efecto de los factores de luz, humedad y fertilidad $(F=93.85, P=<0.01$; $F=15, P=<0.01 ; F=109.06, P=<0.01$; respectivamente). El C:R/V en la radiación alta, humedad alta y fertilidad baja, aumentó $31 \%, 14 \%$ y $32 \%$, respectivamente; en comparación con los tratamientos de radiación baja, humedad baja y fertilidad alta (Cuadro 2). También el C:R/V en la radiación alta y humedad baja aumentó $41 \%$, al compararlo con el de la radiación baja y humedad alta. En el tratamiento de humedad y fertilidad bajas aumentó $44 \%$, al contrastarlo con el de humedad y fertilidad altas. Además, al combinarse la radiación alta, humedad baja y fertilidad baja, dicha variable aumentó $96 \%$; en contraste con el de luz baja, humedad alta y fertilidad alta (Cuadro 6).

Para Opuntia jaliscana la fertilidad baja aumentó el C: $\mathrm{R} / \mathrm{V}$ en $56 \%$, en contraste con la fertilidad alta $(F=49.3$, $P=<0.01)$, los niveles de radiación y humedad no presentaron diferencias (Cuadro 2). También en la radiación baja, humedad baja y fertilidad baja, el C:R/V aumentó 66\%; en comparación con el de radiación baja, humedad baja y fertilidad alta (Cuadro 6). El análisis en O. streptacantha reveló que en la humedad alta y en la fertilidad alta el C:R/V se incrementó en $12.7 \%$ y $10 \%$, correspondientemente, comparados con la humedad y la fertilidad bajas $(F=14.07$, $P=<0.01 ; F=8.54, P=<0.01$; respectivamente, Cuadro 2). También el efecto de la humedad fue distinto por el nivel de radiación: en el tratamiento de radiación baja y humedad alta el C:R/V aumentó 18\%, comparado con el de radiación alta y humedad baja. Mientras que en de radiación y fertilidad altas, ésta variable disminuyó 23.5\% (Cuadro 6).

Análisis de la fluorescencia de la clorofila. La eficiencia fotosintética potencial (EFP) $F_{v} / F_{m}$, de todas las especies en promedio presentó valores de 0.8 . La eficiencia fotosintética actual (EFA), la tasa de transporte de electrones (TTE) y la densidad del flujo de fotones de la fotosíntesis (DFFF), presentaron efecto significativo en el factor radiación $(F=44.25$, $P<0.01)$. La EFA en el nivel alto de radiación fue $11 \%$ mayor que en el bajo $(F=39.79, P<0.01 ; 0.64 \pm 0.01$, $0.71 \pm 0.01$, respectivamente). La TTE fue $59 \%$ mayor en la radiación alta que en la baja $(F=86.6, P<<0.01$; $87.3 \pm 5.3,36.3 \pm 2.6$, respectivamente)

Las protegidas presentaron $21 \%$ mayor EFA que las nodrizas $(F=7.23, P<0.01$; Figura 1$)$. Además, el nivel de radiación afectó la EFA de las especies $(F=2.67, P=0.02)$. Las protegidas exhibieron los valores más altos en ambos niveles de radiación, mientras que las nodrizas disminuyeron su EFA en presencia de radiación alta. Dentro de estas últimas, Acacia farnesiana y Prosopis laevigata fueron las 


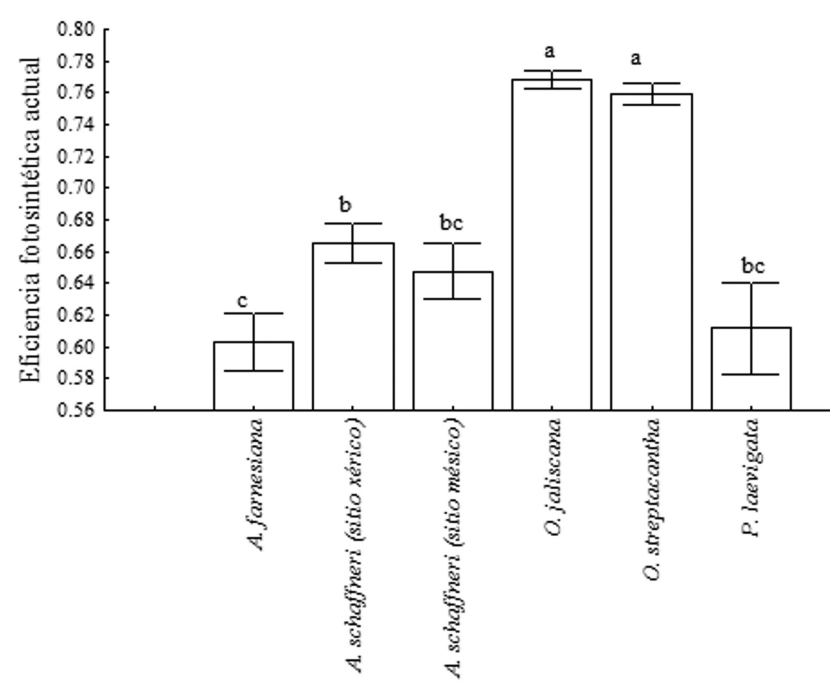

Figura 1. Promedio ( \pm EE) de la eficiencia fotosintética actual, en tres especies nodrizas (Acacia farnesiana, Acacia schaffneri, Prosopis laevigata), y dos protegidas (Opuntia jaliscana, Opuntia streptacantha) $(\mathrm{n}=3)$.

más afectadas al presentar los valores más bajos. La DFFF se presentó de manera diferente en las especies $(F=4.13$, $P<0.01)$ : las nodrizas mostraron mayor respuesta que las protegidas a los flujos de fotones más altos. La humedad afectó de manera diferente la TTE de las especies $(F=259.9$, $P<0.05)$. Opuntia jaliscana disminuyó su TTE en el nivel más bajo de humedad; mientras que A. farnesiana presentó la TTE más alta en el mismo tratamiento.

El ANOVA por especie reveló que la EFA aumentó por efecto de la radiación alta en la mayoría de las especies; los niveles de humedad y fertilidad no afectaron esta variable. Sólo las protegidas evidenciaron respuesta significativa en las interacciones radiación y humedad; así como radiación y fertilidad. En Opuntia jaliscana el tratamiento de radiación baja y humedad alta, y en el de radiación baja y fertilidad alta, incrementaron la EFA $(F=11.78, P<0.01$; $F=5.67, P=0.03$, respectivamente). Opuntia streptacantha no presentó diferencias entre los niveles de fertilidad ni de radiación; sin embargo, en este último factor se observó una tendencia para incrementar la EFA en la radiación baja. Así mismo, se encontró que la humedad alta incrementó la EFA en contraste con la humedad baja $(F=10.7, P<0.01)$.

En todas las especies, la TTE aumentó cuando aumentó el nivel de radiación (Figura 2). Las especies con menor TTE por efecto de la humedad fueron Prosopis laevigata y Acacia farnesiana (TTE respectivo de 1.02 y 1.05 veces más grande en el nivel bajo de humedad que en el nivel alto). Fue notable la diferencia entre el desempeño de A. schaffneri del sitio mésico respecto a la TTE de $A$. schaffneri del sitio xérico, su procedencia determinó una respuesta en TTE en los niveles de humedad. En el sitio xérico la TTE fue mayor en nivel bajo de humedad, mientras que en la del sitio mésico fue en la humedad alta. Para las dos especies de Opuntia la TTE fue mayor (entre 1.8 y 2.5 veces) en la humedad alta (60\%).

\section{Discusión}

Tasa relativa de crecimiento (TRC). Se esperaba que las protegidas presentaran tasas relativas de crecimiento más
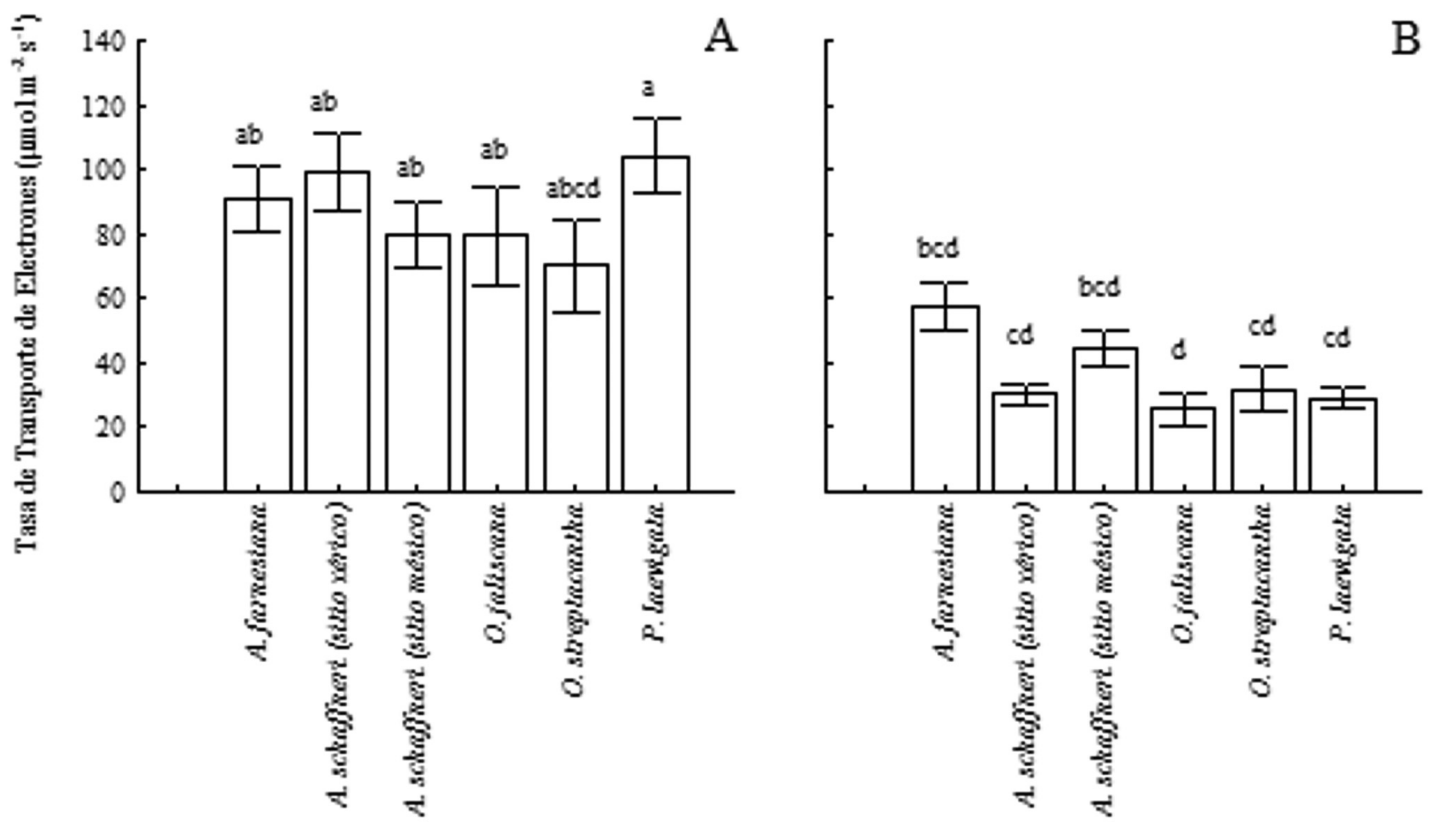

Figura 2. Promedio (+ EE) de la tasa de transporte de electrones de tres nodrizas (Acacia farnesiana, Acacia schaffneri, Prosopis laevigata), y dos protegidas (Opuntia jaliscana, Opuntia streptacantha), en dos condiciones de radiación: A (Radiación alta) y B (Radiación Baja) $(\mathrm{n}=3)$. 
altas en la radiación baja, mientras que en las nodrizas el crecimiento fuera mayor en la radiación alta. Sin embargo, la mayoría de las especies presentaron tasas relativas de crecimiento mayores en la radiación alta que en la baja. Además, se esperaba que las nodrizas crecieran más que las protegidas por efecto de la radiación alta; sin embargo, se encontraron claras diferencias en el crecimiento entre protegidas y nodrizas; las primeras crecieron entre 5\% y $27 \%$ más que las segundas. Este resultado es contrario a la hipótesis que plantea que las especies protegidas, que son especies con crecimiento lento (Grime, 1977), presentan TRC más bajas que las especies nodrizas (sensu Grime y Hunt, 1975), especies de crecimiento rápido (Lambers y Poorter, 1992; Chapin et al., 1993).

Por otra parte, el efecto positivo que tiene la nodriza sobre la supervivencia y crecimiento de las plántulas de especies protegidas por la reducción de la radiación ha sido bien documentado (Valiente-Banuet y Ezcurra, 1991; Flores-Martínez et al., 1994). Sin embargo, en este trabajo se encontró que las protegidas crecieron más en condiciones de luz alta; estos resultados son contrarios con otros estudios que mencionan que la reducción en la radiación aumenta la supervivencia de las protegidas y tiene un efecto positivo en su crecimiento (Valiente-Banuet y Ezcurra, 1991; FloresMartínez et al., 1994; Palleiro et al., 2006), pero concuerda con la expectativa de que radiaciones altas resultan en TRC altas (Martínez-Berdeja y Valverde 2008; Miquelajauregui y Valverde, 2010).

Se considera que bajo el dosel de las nodrizas, las protegidas incrementan su supervivencia y crecimiento como resultado de una mayor disponibilidad de humedad (Valiente-Banuet y Ezcurra, 1991; Flores-Martínez et al., 1994; Martínez-Berdeja y Valverde, 2008; Miquelajauregui y Valverde, 2010). En este trabajo se encontró que la humedad alta aumentó el crecimiento en la mayoría de las especies. Sin embargo, sólo en las protegidas se encontró que la interacción radiación alta y humedad alta afectó positivamente el crecimiento; este resultado está de acuerdo con los obtenidos por Miquelajauregui y Valverde (2010) en cactáceas columnares.

Por efecto de la fertilidad alta (suelo debajo de dosel de Acacia schaffneri), las suculentas crecieron entre $23.5 \%$ y $30.4 \%$ más que las leñosas; de hecho, éstas últimas disminuyeron su crecimiento en el suelo de dosel. El mayor crecimiento de las suculentas coincide con la hipótesis que plantea que el aumento en fertilidad debajo del dosel de plantas adultas crea "islas de fertilidad" que facilitan y aumentan el crecimiento de plántulas suculentas que crecen bajo su sombra (García-Moya y McKell, 1970; Franco y Nobel, 1989; Valiente-Banuet et al., 1991). También coincide con lo reportado por Castillo et al. (2010) quienes encontraron que la supervivencia y las tasas de crecimiento del cactus columnar Neobuxbaumia mezcalaensis fueron altas y positivamente relacionadas con el incremento de la distancia filogenética a diferentes especies nodrizas en el valle de Tehuacán-Cuica- tlán; es decir, el efecto nodriza es mayor cuando crece bajo el dosel de especies no emparentadas.

El menor crecimiento de los arbustos leñosos en el suelo obtenido debajo de dosel de Acacia schaffneri coincide con Puerta-Piñero et al. (2006), quienes encontraron que las plántulas de Quercus ilex crecieron menos en suelo con mayor N, P y K obtenido debajo de individuos adultos de Q. ilex (considerados como plantas nodriza), que en suelos de espacios abiertos con menor cantidad de nutrientes. Es probable que los suelos debajo de A. schaffneri y de $Q$. ilex contengan patógenos que inhiban el crecimiento de las plántulas de sus mismas especies y de otras arbustivas emparentadas, lo anterior basado en Webb et al. (2006), quienes encontraron que los patógenos pueden infectar hospederos emparentados debido a que las barreras contra patógenos son con frecuencia filogenéticamente conservadas.

Se puede suponer, que la acción benéfica de la sombra del dosel de las leñosas sobre las suculentas está mediada por la variación temporal que presenta la precipitación (Tielbörger y Kadmon, 2000), cuando en los sitios abiertos hay déficit de humedad; sin embargo, en los periodos con lluvias abundantes la acción benéfica del dosel se convierte en una limitante de acceso a la luz en las protegidas (Franco y Nobel, 1989), por lo que en ese caso la interacción sería de competencia más que de facilitación (Holmgren et al., 1997).

Otra consideración importante es que las suculentas al presentar mayor crecimiento que las nodrizas, pueden resultar en una rápida ocupación del espacio y por lo tanto, sea una ventaja competitiva (Grime y Hunt, 1975), como lo encontraron Flores y Yeaton (2000) en Opuntia streptacantha, que en la fase de plántulas se desempeñan como protegidas y en la fase de adultas se comportan como especies competitivamente superiores y reemplazan a su nodriza (Acacia schaffneri).

Cociente del área fotosintética (CAF). La limitación de luz aumenta la supervivencia de las protegidas (Valiente-Banuet y Ezcurra, 1991; Flores-Martínez et al., 1994), pero, al disminuir la luz, también disminuye su crecimiento (Sack, 2004). Las plantas que crecen en ambientes sombreados responden a esta condición asignando más biomasa a los órganos fotosintéticos para incrementar el área de exposición y adquirir más luz (Villar et al., 2008); es decir, presentan un CAF alto, lo que maximiza la intercepción de luz (Poorter y Van der Werf, 1998; Quero et al., 2008). Entonces la asignación de recursos al área fotosintética se convierte en un factor clave en la eficiencia para cosechar luz en las plántulas que crecen debajo del dosel de nodrizas (Bazzaz y Wayne, 1994). En este estudio todas las especies presentaron un CAF mayor en la radiación baja que en la alta; estos resultados son consistentes con los encontrados en otros trabajos (Walters et al., 1993; Poorter et al., 1995; Reich et al., 1998; Walters y Reich, 1999; Sánchez-Gómez et al., 2006; Portsmuth y Niinemets, 2007). Sin embargo, en comparación con 
las protegidas, las nodrizas realizaron menos ajustes morfológicos (tuvieron menor $\mathrm{CAF}$ ) en el nivel bajo de radiación. Las nodrizas presentaron valores del CAF hasta dos veces menores que las protegidas, por lo que las segundas fueron más eficientes en la captura de luz bajo condiciones de radiación baja (Poorter, 1999).

Tasa de asimilación neta (TAN). En todas las especies la TAN fue mayor en la radiación alta que en la baja. Sin embargo, las nodrizas expusieron valores de la TAN hasta dos veces mayores que las protegidas. Una TAN alta es vital para especies que crecen en espacios abiertos con demandas de luz altas, donde son importantes las adaptaciones fisiológicas que les ayudan a equilibrar la capacidad fotosintética y los gastos en la respiración (Strauss-Debenedetti y Bazzaz, 1991; Montgomery, 2004). Esta es una característica fisiológica de especies adaptadas a niveles de radiación altos (Pons, 1977; Valladares et al., 2002; Souza y Válio, 2003; Montgomery, 2004; Portsmuth y Niinemets, 2007). Además de la luz, la fertilidad fue clave en las diferencias fisiológicas entre especies. En la mayoría de las nodrizas, el suelo de fertilidad baja incrementó la TAN, mientras que en las protegidas no fueron afectadas. Esta condición ya ha sido encontrada en especies que se establecen en espacios abiertos con suelos pobres, como en la sabana brasileña (Hoffmann y Franco, 2003).

Las nodrizas presentaron mayores cambios debidos al componente fisiológico (TAN) y, en las protegidas fue el componente morfológico (CAF). Estos resultados están de acuerdo con otros autores (Poorter y Van der Werf, 1998; Lambers et al., 1998; Veneklaas y Poorter, 1998; Montgomery, 2004), en que la TAN tiene una contribución importante con variación de la TRC de especies que crecen en intensidades altas de luz, mientras que el CAF es el componente que más contribuye a la variación de la TRC en ambientes sombreados. El crecimiento de las nodrizas presentó una correlación positiva con el componente fisiológico (TAN); mientras que en las protegidas fue negativa y ambos componentes (CAF y TAN), contribuyeron en la variación de la tasa relativa de crecimiento. Este balance puede indicar que las protegidas se adaptan tanto a ambientes sombreados como iluminados (i.e. diferentes condiciones de radiación solar), por lo que pueden explotar mayor cantidad de recursos (Poorter y Garnier, 1999; Portsmuth y Niinemets, 2007). Es probable que estas especies, en la fase de plántula, se comporten más como especies tolerantes a la sombra que como especies adaptadas a la sombra (Valladares et al., 2002; Niinemets y Valladares, 2004). Además, presentaron mayor adaptación a suelos ricos en nutrimentos, una característica importante en especies que aumentan su cosecha de luz (mayor CAF), al establecerse bajo el dosel de otras especies (Portsmuth y Niinemets, 2006).

Cociente raíz/vástago $(\mathrm{C}: \mathrm{R} / \mathrm{V})$. Se encontró una clara tendencia en la mayoría de las especies en asignar más biomasa a la raíz en el suelo de menor fertilidad. Estos resultados son compatibles con otras observaciones (Huante et al., 1995; Portsmuth y Niinemets, 2007; Villar et al., 2008). En contraste con las protegidas, las nodrizas presentaron los valores más altos del cociente raíz/vástago $(\mathrm{C}: \mathrm{R} / \mathrm{V})$. Un $\mathrm{C}: \mathrm{R} / \mathrm{V}$ alto indica que las plantas asignan más biomasa a la raíz, lo que maximiza la toma de nutrimentos en suelos con fertilidad limitada (Tilman, 1988). En los espacios abiertos las plantas deben establecer raíces profundas que les asegure la adquisición de agua, espacio, luz y nutrimentos. Desde la perspectiva ecológica, las plantas con mayor proporción de biomasa en la raíz tienen un crecimiento más lento; sin embargo, obtienen ventajas como una mayor superficie de absorción y mayor supervivencia en hábitats donde los recursos como el agua y los nutrimentos son limitados (Villar et al., 2008).

Fluorescencia de la clorofila. Las reacciones fotoquímicas de la fotosíntesis son sensibles a la radiación alta, lo que afecta la eficiencia fotosintética potencial y actual del fotosistema II (Osmond, 1994), por otro lado, a la reducción de la capacidad fotoquímica total se le conoce como fotoinhibición (Ribeiro et al., 2005). La eficiencia fotosintética potencial (EFP) de una planta saludable presenta valores de 0.8 , y valores menores indican estrés en plantas por exceso de luz (Cavender-Bares y Bazzaz, 2004). En este trabajo, los valores de EFP oscilaron alrededor de 0.8 , por lo que ninguna especie presentó fotoinhibición como respuesta a la radiación alta (Cavender-Bares y Bazzaz, 2004).

La densidad de flujo de fotones para la fotosíntesis (DFFF) de los niveles de luz afectó la eficiencia fotosintética actual (EFA) y la tasa de transporte de electrones (TTE). Las nodrizas presentaron menor EFA que las protegidas en el tratamiento de luz alta. Estos resultados están de acuerdo con los encontrados en especies que crecen en sitios abiertos y debajo de arbustos (Souza et al., 2009). Frecuentemente, especies arbóreas adaptadas a espacios abiertos presentan menor EFA en contraste con especies de ambientes sombreados (Ribeiro et al., 2005). Además, en este estudio, las protegidas mostraron valores de EFA superiores a los encontrados en otras protegidas (Hernández-González y Briones, 2007).

Los valores de la tasa de transporte de electrones (TTE) más altos fueron encontrados en condiciones de irradiación alta, y como se esperaba, las nodrizas presentaron TTE mayor que las protegidas. Estos resultados son similares a los encontrados en especies de árboles tropicales de diferentes estadios sucesionales (Ribeiro et al., 2005). Por otra parte, en Opuntia jaliscana la humedad baja fue la que más disminuyó la TTE. Se conoce que el estrés hídrico aumenta la competencia por el uso de los electrones, entre el proceso de fotorespiración y la asimilación de $\mathrm{CO}_{2}$ (Flexas et al., 1999); estos resultados coinciden con los encontrados por Flexas et al. (2002) en plantas $\mathrm{C}_{3}$ en condiciones de campo. 


\section{Conclusiones}

En este trabajo es evidente que las nodrizas presentaron características morfológicas y fisiológicas de especies adaptadas a sitios abiertos y soleados, donde prevalecen condiciones limitantes de humedad y fertilidad; mientras que las suculentas presentaron características de especies protegidas, adaptadas a la sombra, con humedad y fertilidad altas. Las diferentes respuestas ecofisiológicas, entre nodrizas y protegidas, sugieren que pueden tener capacidad competitiva contrastante, y la segregación de su nicho de regeneración está relacionada con la disponibilidad de recursos. Tales diferencias pueden desempeñar un papel importante en el éxito de la coexistencia entre estas especies que forman interacciones de facilitación, lo que organiza la dinámica de poblaciones en comunidades vegetales del Altiplano Mexicano.

\section{Agradecimientos}

Agradecemos el apoyo al Consejo Nacional para la Ciencia y la Tecnología, al Programa Multidisciplinario de Posgrado en Ciencias Ambientales de la Universidad Autónoma de San Luis Potosí, al Instituto de Investigación en Zonas Desérticas, al Instituto Potosino de Investigación Científica y Tecnológica, al Dr. Pablo Delgado y a la Dra. Olivia Hernández por su apoyo para las mediciones y análisis de la fluorescencia de la clorofila II, a Ruth Mireles, Miguel Cortina, Yakanqui Romo y Jaciel Flores por su ayuda para la toma de datos, a Oscar Carvajal por su apoyo en la conversión de las figuras, a Edgar López por su revisión en la traducción del resumen en inglés, así como a dos revisores anónimos por sus comentarios y sugerencias que ayudaron a mejorar el escrito.

\section{Literatura citada}

Barnes P.W. y Archer S. 1999. Tree-shrub interactions in subtropical savanna parkland: competition or facilitation? Journal of Vegetation Science 10:525-536.

Brown J.R. y Archer S. 1987. Woody plant seed dispersal and gap formation in a North American subtropical savanna: the role of domestic herbivores. Vegetatio 73:73-80.

Bush J.K. y Van Auken O.W. 1987. Light requirements for growth of Prosopis glandulosa seedlings. Southwestern Naturalist 32:469-473.

Butterfield B.J. y Briggs J.M. 2011. Regeneration niche differentiates functional strategies of desert woody plant species. Oecologia 165:477-487.

Callaway R.M. 1995. Positive interactions among plants. The Botanical Review 61:306-349.

Cardillo E. y Bernal C.J. 2006. Morphological response and growth of cork oak (Quercus suber L.) seedlings at different shade levels. Forest Ecology and Management 222:296-301.

Castillo J.P., Verdú M. y Valiente-Banuet A. 2010. Neighborhood phylodiversity affects plant performance. Ecology 91:3656-3663.
Cavender-Bares J. y Bazzaz F.A. 2004. From leaves to ecosystems: Using chlorophyll fluorescence to assess photosynthesis and plant function in ecological studies. En: Papageorgiou G.C. y Govindjee. Eds. Chlorophyll a Fluorescence: A Signature of Photosynthesis, pp. 737-755, Springer, Dordrecht.

Chapin III F.S., Moilanen L. y Kielland K. 1993. Preferential use of organic nitrogen for growth by a non-mycorrhizal arctic sedge. Nature 361:150-153.

Cody M.L. 1993. Do cholla cacti (Opuntia spp., subgenus Cylindropuntia) use or need nurse plants in the Mojave Desert? Journal of Arid Environments 24:139-154.

Domingo F., Villagarcía L., Brenner A.J. y Puigdefábregas J. 1999. Evapotranspiration model for semi-arid shrub-lands tested against data from SE Spain. Agricultural and Forest Meteoro$\log y$ 95:67-84.

Ehleringer J. 1981. Leaf absorptances of Mohave and Sonoran Deserts plants. Oecologia 49:366-370.

Evans G.C. 1972. The Quantitative Analysis of Plant growth. University of California Press, Berkeley.

Flexas J., Escalona J.M. y Medrano H.1999. Water stress induces different levels of photosynthesis and electron transport rate regulation in grapevines. Plant, Cell and Environment 22:39-48.

Flexas J., Escalona J.M., Evain S., Gulías J., Moya I., Osmond C.B. y Medrano H. 2002. Steady-state chlorophyll fluorescence (Fs) measurements as a tool to follow variations of net $\mathrm{CO}_{2}$ assimilation and stomatal conductance during water-stress in $\mathrm{C}_{3}$ plants. Physiologia Plantarum 114:231-240.

Flores J. y Jurado E. 2003. Are nurse-protégé interactions more common among plants from arid environments? Journal of Vegetation Science 14:911-916.

Flores-Martínez A., Ezcurra E. y Sánchez-Colón S. 1994. Effect on Neobuxbaumia tetetzo on growth and fecundity of its nurse plant Mimosa luisana. Journal of Ecology 82:325-330.

Flores F.J.L y Yeaton H.R.I. 2000. La importancia de la competencia en la organización de las comunidades vegetales en el Altiplano Mexicano. Interciencia 25:365-371.

Franco A.C. y Nobel P.S.1989. Effect of nurse plants on the microhabitat and growth of cacti. Journal of Ecology 77:870-886.

García-Moya E. y McKell C.M. 1970. Contribution of shrubs to the nitrogen economy of a desert-wash plant community. Eco$\operatorname{logy}$ 51:81-88.

Godínez-Álvarez H., Valverde T. y Ortega-Baes P. 2003. Demographic trends in the Cactaceae. The Botanical Review 69:173-203.

Gonçalvez J.F.C., Barreto D.C.S., Santos Junior U.M., Fernandes A.V., Sampaio P.T.B. y Buckeridge M.S. 2005. Growth, photosynthesis and stress indicators in young rosewood plants (Aniba rosaeodora Ducke) under different light intensities. Brazilian Journal of Plant Physiology 17:325-334.

Grime J.P. 1977. Evidence for the existence of three primary strategies in plants and its relevance to ecological and evolutionary theory. American Naturalist 111:1169-1194.

Grime J.P. y Hunt R. 1975. Relative growth-rate; its range and adaptative significance in a local flora. Journal of Ecology 63:393-422.

Hernández-González O. y Briones V.O. 2007. Crassulacean acid metabolism photosynthesis in columnar cactus seedlings during ontogeny: the effect of light on nocturnal acidity accumulation and chlorophyll fluorescence. American Journal of Botany 94:1344-1351. 
Hoffmann W.A. y Franco A.C. 2003. Comparative growth analysis of tropical forest and savanna woody plants using phylogenetically independent contrasts. Journal of Ecology 91:475-484.

Holmgren M., Scheffer M. y Huston M.A. 1997. The interplay of facilitation and competition in plant communities. Ecology 78:1966-1975.

Huante P., Rincón E. y Chapin III F.S. 1995. Responses to phosphorus of contrasting successional tree-seedling species from the tropical deciduous forest of Mexico. Functional Ecology 9:760-766.

INEGI (Instituto Nacional de Estadística, Geografía e Informática). 1981. Síntesis geográfica del estado de Jalisco. Secretaría de Planeación y Presupuesto. México, D.F.

INEGI (Instituto Nacional de Estadística, Geografía e Informática). 2002. Síntesis geográfica del estado de San Luis Potosí. 2da. edición. Secretaría de Planeación y Presupuesto, México. D.F.

Jandel Scientific. 1993. Sigma Scan/Image. Measurement Software for Windows. Jandel Scientific, San Rafael.

Lambers H. y Poorter H. 1992. Inherent variation in growth rate between higher plants: A search for physiological causes and ecological consequences. Advances in Ecology Research 23:187-261.

Lambers H., Chapin III F.S. y Pons T.L. 1998. Plant Physiological Ecology. Springer-Verlag, Nueva York.

Martínez-Berdeja A. y Valverde T. 2008. Growth response of three globose cacti to radiation and soil moisture: An experimental test of the mechanism behind the nurse effect. Journal of Arid Environments 72:1766-1774.

Maxwell K. y Johnson G.N. 2000. Chlorophyll fluorescence - a practical guide. Journal of Experimental Botany 51:659-668.

McAuliffe J.R. 1988. Markovian dynamics of simple and complex desert plant communities. American Naturalist 131: 459-490.

Miquelajauregui Y. y Valverde T. 2010. Survival and early growth of two congeneric cacti that differ in their level of rarity. Journal of Arid Environments 74:1624-1631.

Miranda F. y Hernández X.E. 1963. Los tipos de vegetación de México y su clasificación. Boletín de la Sociedad Botánica de México 28:29-179.

Montgomery R. 2004. Relative importance of photosynthetic physiology and biomass allocation for tree seedling growth across a broad light gradient. Tree Physiology 24:155-167.

Niering W.A., Whittaker R.H. y Lowe C.H. 1963. The saguaro: a population in relation to environment. Science 142:15-23.

Niinemets Ü. y Valladares F. 2004. Photosynthetic acclimation to simultaneous and interacting environmental stresses along natural light gradients: optimality and constraints. Plant Biology 6:254-268.

Nobel P.S. 1980. Morphology, nurse plants and minimum apical temperatures for young Carnegiea gigantea. Botanical Gazette 141:188-191.

Osmond C.B. 1994. What is photoinhibition? Some insights from comparisons of shade and sun plants. En: Baker N.R. y Bowyer J.R. Eds. Photoinhibition: Molecular Mechanisms to the Field, pp. 1-24, Bios Scientific Publishers, Oxford.

Palleiro N., Mandujano M.C. y Golubov J. 2006. Aborted fruits of Opuntia microdasys (Cactaceae): insurance against reproductive failure. American Journal of Botany 93:505-511.

Pons T.L. 1977. An ecophysiological study in the field layer of ash coppice II. Experiment with Geum urbanum and Cirsium pa- lustre in different light intensities. Acta Botanica Neerlandica 26:29-42.

Poorter L. 1999. Growth responses of 15 rain-forest tree species to a light gradient: the relative importance of morphological and physiological traits. Functional Ecology 13:396-410.

Poorter H. y Garnier E. 1999. Ecological significance of relative growth rate and its components. En: Pugnaire F.I. y Valladares F. Eds. Handbook of Functional Plant Ecology, pp. 81-120, CRC Press, Nueva York.

Poorter H. y Van der Werf A.A. 1998. Is inherent variation in RGR determined by LAR at low irradiance and by NAR at high irradiance? A review of herbaceous species. En: Lambers H., Poorter H. y van Vuuren M.M.I. Eds. Inherent Variation in Plant Growth. Physiological Mechanisms and Ecological Consequences, pp 309-336, Backhuys Publishers, Leiden.

Poorter H., van de Vijver C.A.D.M., Boot R.G.A. y Lambers H. 1995. Growth and carbon economy of a fast-growing and a slow-growing grass species as dependent on nitrate supply. Plant and Soil 171:217-227.

Portsmuth A. y Niinemets Ü. 2006. Interacting controls by light availability and nutrient supply on biomass allocation and growth of Betula pendula and B. pubescens seedling. Forest Ecology and Management 227:122-134.

Portsmuth A. y Niinemets Ü. 2007. Structural and physiological plasticity in response to light and nutrients in five temperate deciduous woody species of contrasting shade tolerance. Functional Ecology 21:61-77.

Puerta-Piñero C., Gómez J.M. y Zamora R. 2006. Species-specific effects on topsoil development affect Quercus ilex seedling performance. Acta Oecologica 29:65-71.

Quero J.L., Villar R., Marañón T., Zamora R., Vega D. y Sack L. 2008. Relating leaf photosynthetic rate to whole-plant growth: drought and shade effects on seedlings of four Quercus species. Functional Plant Biology 35:725-737.

Reich P.B., Walters M.B., Tjoelker M.G., Vanderklein D. y Buschena C. 1998. Photosynthesis and respiration rates depend on leaf and root morphology and nitrogen concentration in nine boreal tree species differing in relative growth rate. Functional Ecology 12:395-405.

Ribeiro R.V., Souza G.M., Oliveira R.F. y Machado E.C. 2005. Photosynthetic responses of tropical tree species from different successional groups under contrasting irradiance conditions. Revista Brasileira de Botânica 28:149-161.

Rzedowski J. 1966. Vegetación del estado de San Luis Potosí, México. Acta Científica Potosina 5:5-291.

Sack L. 2004. Responses of temperate woody seedlings to shade and drought: do trade-offs limit potential niche differentiation? Oikos 107:110-127.

Sánchez-Gómez D., Valladares F. y Zavala M.A. 2006. Functional traits and plasticity in response to light in seedlings of four Iberian forest tree species. Tree Physiology 26:1425-1433.

SAS Institute Inc. 2002. SAS/STAT software, version 9. SAS Institute, Inc., Cary.

Souza R.P. y Válio I.F.M. 2003. Seedling growth of fifteen Brazilian tropical tree species differing in successional status. Revista Brasileira de Botânica 26:35-47.

Souza G.M., Balmant B.D., Vítolo H.F., Gomes K.B.P., Florentino T.M., Catuchi T.A. y Vieira W.L. 2009. Estratégias de utilização de luz e estabilidade do desenvolvimento de plântulas de Cordia superba Cham. (Boraginaceae) crescidas em diferentes am- 
bientes luminosos. Acta Botanica Brasilica 23:474-485.

Strauss-Debenedetti S. y Bazzaz F.A. 1991. Plasticity and acclimation to light in tropical Moraceae of different successional positions. Oecologia 87:377-387.

Tielbörger K. y Kadmon R. 2000. Temporal environmental variation tips the balance between facilitation and interference in desert plants. Ecology 81:1544-1553.

Tilman D. 1988. Plant Strategies and the Dynamics and Structure of Plant Communities. Princeton University Press, Nueva Jersey.

Tirado R. y Pugnaire F.I. 2003. Shrub spatial aggregation and consequences for reproductive success. Oecologia 136:296-301.

Valiente-Banuet A. y Ezcurra E. 1991. Shade as cause of the association between the cactus Neobuxbaumia tetetzo and the nurse plant Mimosa luisana in the Tehuacán Valley, México. Journal of Ecology 79:961-971.

Valiente-Banuet A. y Verdú M. 2007. Facilitation can increase the phylogenetic diversity of plant communities. Ecology Letters 10:1029-1036.

Valiente-Banuet A., Vite F. y Zavala-Hurtado J.A. 1991. Interaction between the cactus Neobuxbaumia tetetzo and the nurse shrub Mimosa luisana. Journal of Vegetation Science 2:11-14.

Valladares F., Skillman J.B. y Pearcy R.W. 2002. Convergence in light capture efficiencies among tropical forest understory plants with contrasting crown architectures: a case of morphological compensation. American Journal of Botany 89:1275-1284.

Veneklaas E.J. y Poorter L. 1998. Growth and carbon partitioning of tropical tree seedlings in contrasting light environments. En: Lambers H, Poorter H. y van Vuuren M.M.I. Eds. Inherent Variation in Plant Growth, pp. 337-361, Backhuys Publishers, Leiden.

Villar R., Ruiz-Robleto J,. Quero J.L., Poorter H., Valladares F. y Marañon T. 2008. Tasas de crecimiento en especies leñosas: aspectos funcionales e implicaciones ecológicas. En: Valladares F. Ed. Ecología del Bosque Mediterráneo en un Mundo Cambiante, pp. 191-227, Ministerio de Medio Ambiente, EGRAF, S.A., Madrid.

Walters M.B. y Reich P.B. 1999. Low-light carbon balance and shade tolerance in the seedlings of woody plants: do winter deciduous and broad-leaved evergreen species differ? New Phytologist 143:143-154.

Walters M.B., Kruger E.L. y Reich P.B. 1993. Growth, biomass distribution and $\mathrm{CO}_{2}$ exchange of northern hardwood seedlings in high and low light: relationships with successional status and shade tolerance. Oecologia 94:7-16.

Webb C.O., Gilbert G.S., y Donoghue M.J. 2006. Phylodiversitydependent seedling mortality, size structure, and disease in a Bornean rain forest. Ecology 87(Suppl.):S123-S131.

Recibido: 8 de octubre de 2012

Aceptado: 29 de noviembre de 2012 
